\title{
Review Paper: Literature Review of the Effect of Ankle-Foot Orthosis on Gait Param- eters After Stroke
}

\author{
Elnaz Esfandiari ${ }^{1},{ }^{*}$ Mokhtar Arazpour ${ }^{2}$, Hassan Saeedi ${ }^{1}$, Amir Ahmadi $^{3}$
}

1. Department of Orthotics and Prosthetics, School of Rehabilitation Sciences, Iran University of Medical Sciences, Tehran, Iran. 2. Department of Orthotics and Prosthetics, University of Social Welfare and Rehabilitation Sciences, Tehran, Iran.

3. Department of Physiotherapy, School of Rehabilitation Sciences, Iran University of Medical Sciences, Tehran, Iran.

Received: 20 Oct. 2016 Accepted: 07 Mar. 2017

Keywords:

Stroke, Drop foot Ankle foot orthoses, Gait parameters
Citation: Esfandiari E, Arazpour M, Saeedi H, Ahmadi A. [Literature Review of the Effect of Ankle-Foot Orthosis on Gait Parameters After Stroke (Persian)]. Archives of Rehabilitation. 2017; 18(2):164-179. http://dx.doi.org/10.21859/jrehab-1802160

doi : http://dx.doi.org/10.21859/jrehab-1802160

\begin{abstract}
Objective Stroke occurs when the supply of blood to the brain is either interrupted or reduced. The clinical presentation varies from minor neurological symptoms to severe deficits, depending on the location and the size of the brain lesion. Hemiparesis is one of the most striking features in the acute phase. Many other deficits may also be present, including postural imbalance. All persistent neurological deficits may cause more or less severe activity limitations in several domains of human functioning. Regaining walking ability is a major goal during the rehabilitation of stroke patients. Therefore, using orthoses can be beneficial for them. Ankle-Foot Orthoses (AFOs) is one of the most common therapeutic approaches to control foot drop among stroke patients. AFOs prevent drooping or other unintended movements of the foot and ankle by providing stability in optimum conditions. It also helps in regaining normal walking posture in stroke patients. The aim of this review was to evaluate the efficiency of the AFOs on balance and examine the effectiveness of temporal spatial and kinetic gait kinematics in stroke patients with foot drop. Materials \& Methods Science Direct, Springer, Google Scholar, PubMed, Ovid databases were searched for articles published between 1996 and 2016 of studies on patients with drop foot wearing the AFOs. After reviewing and categorizing the articles, they were analyzed based on spatiotemporal parameters, gait kinetics, gait kinematics and stability. A total of 21 articles were selected for final evaluation. Results Twenty-one articles were analyzed in relation to the effect of the AFOs on gait parameters in stroke patients. Spatiotemporal parameters were evaluated in 14 articles, and kinetics and kinematics parameters were analyzed in seven articles. AFOs have a significant impact on the length and width of the steps but had no significant effect on speed, cadence, symmetry of gait and balance. Also, AFOs improved kinetic parameters of gait, ankle kinematics and kinematics of the knee in the static phase but had no significant effect on knee joint kinematics and kinematics of the hip joint in the frontal and sagittal. There are a few studies with regard to the effects of AFOs on the moment of inertia and joints power, but the results of the present study showed no significant difference in these parameters.

Conclusion This study showed that the AFOs based on the models (static or dynamic) had a paradoxical effect on balance, kinetic and kinematic parameters of gait in the stroke patients. AFOs had a significant improvement in balance, kinetic and kinematic parameters of gait compared to those without orthosis situation in the stroke patients. According to the result of this study, depending on the patient's needs and situation, the best and the most suitable ankle foot orthoses should be designed and custom molded for them.
\end{abstract}

Address: Department of Orthotics and Prosthetics, University of Social Welfare and Rehabilitation Sciences, Tehran, Iran. Tel: +98 (21) 22180010

E-Mail: m.arazpour@yahoo.com 


\title{
مقاله مرورى: بررسى تأثير ارتزهاى ميج يا-يايى بر متغيرهاى راهرفتن و تعادل افراد سكته مغزى
}

\author{
الناز اسفنديارى'، "مختار عراضيورّ، حسن سعيدى'، امير احمدى'

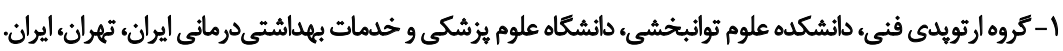

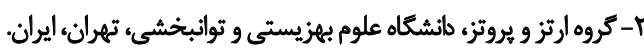

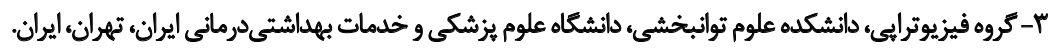

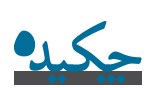

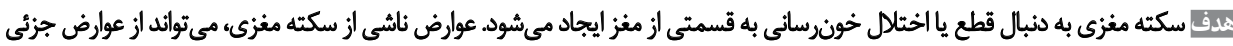

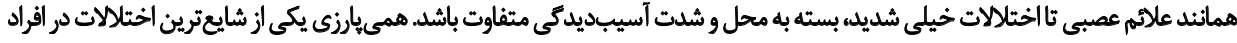

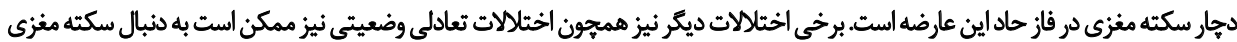

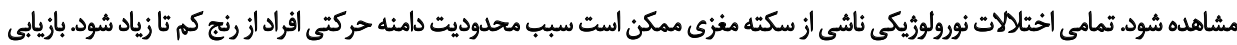

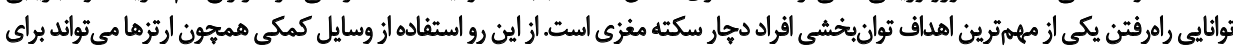

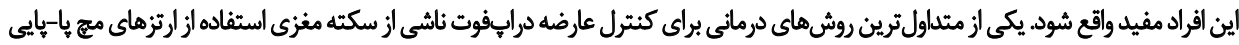

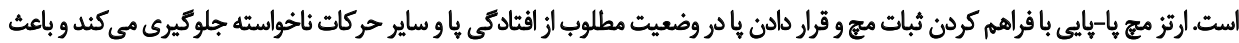

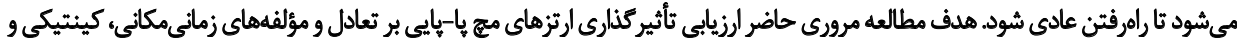

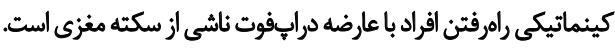

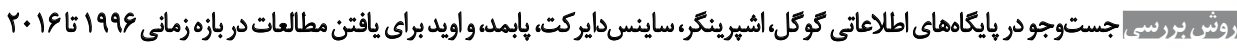

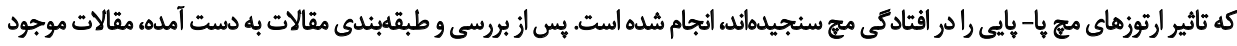

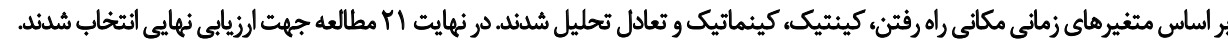

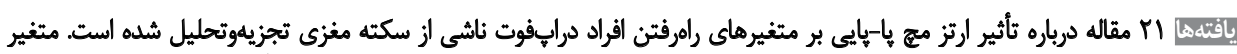

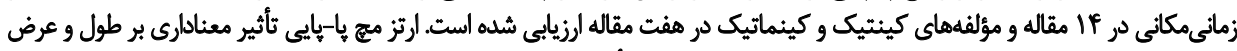

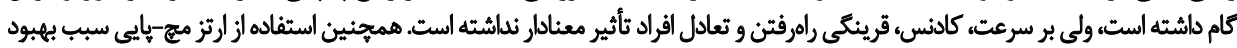

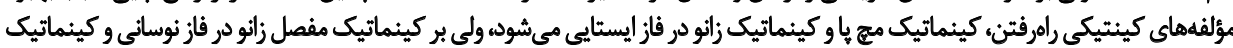

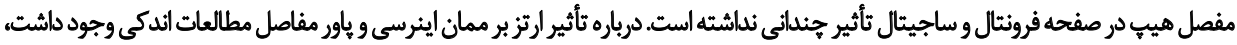

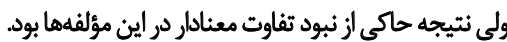

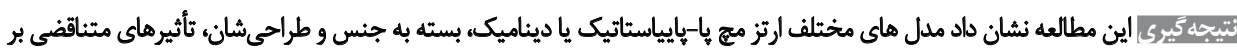

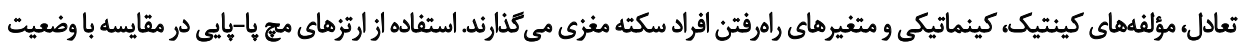

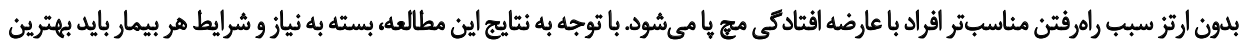

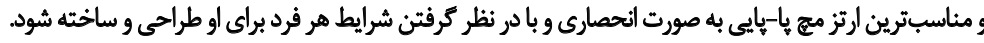

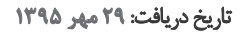

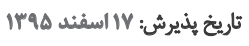

مغزى مي ميرند، توانايىهايى كه توسط اين ناحيه كنترل daleo

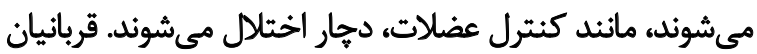

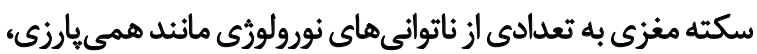

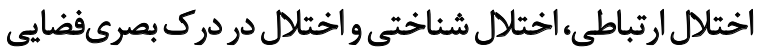

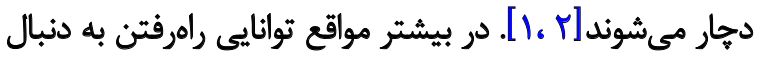

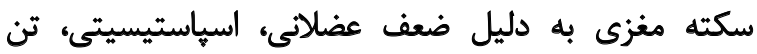
غيرعادى عضلانى، به خطر افتادن كنترل حسى خركتى، نداشتن النئن

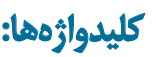

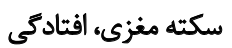

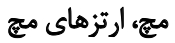

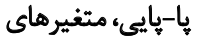

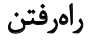

-

سكته مغزى' يكى از علل اصلى ناثوانى و مرك در سراسر

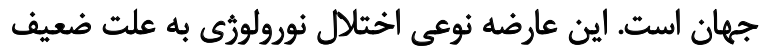

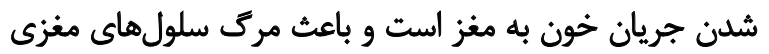

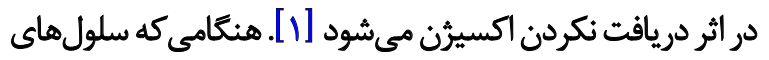

1. Stroke

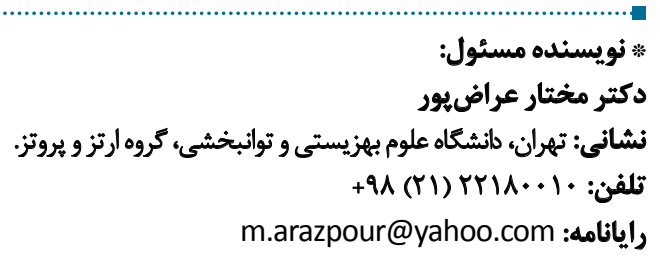




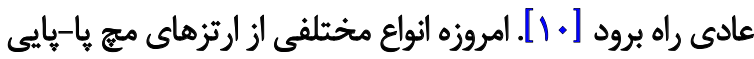

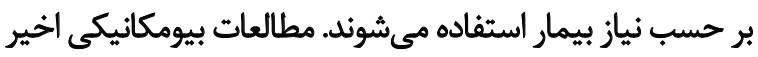

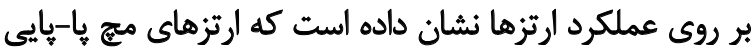

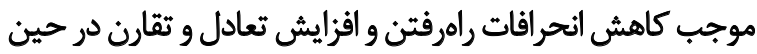

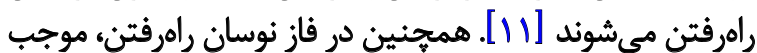

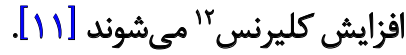

تاكنون جندين مطالعه مرورى در زمينه تأثير انواع مختلف

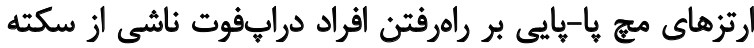

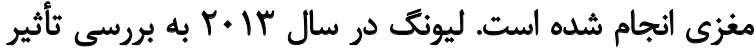

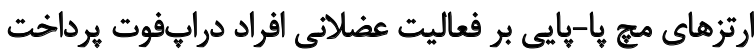

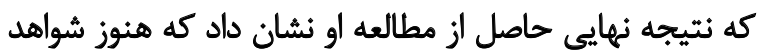

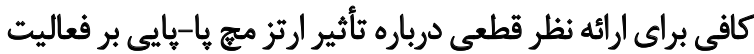

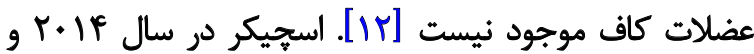

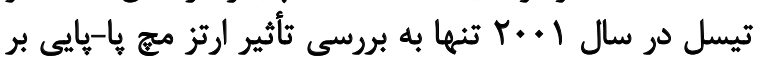

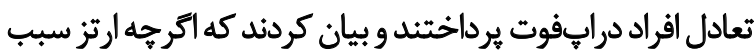

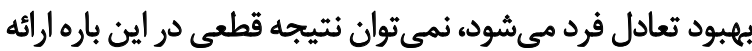

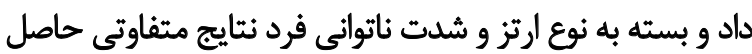

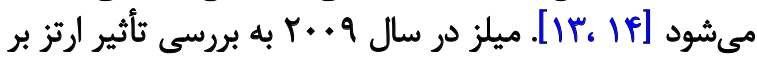

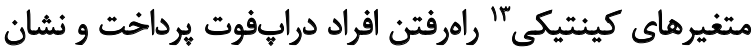

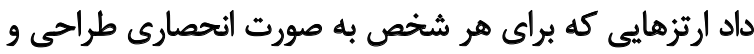

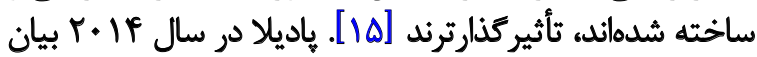

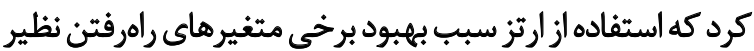

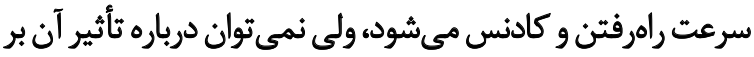

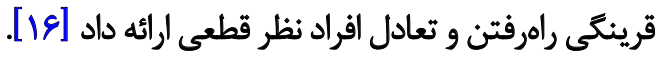

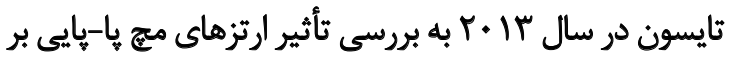

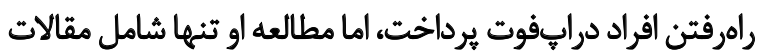

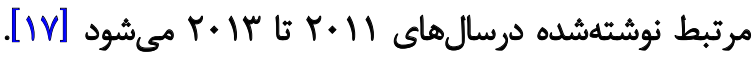

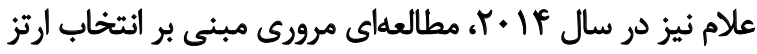

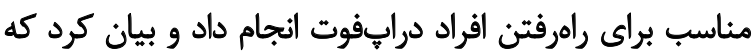

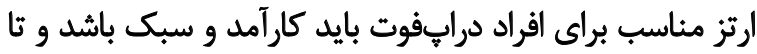

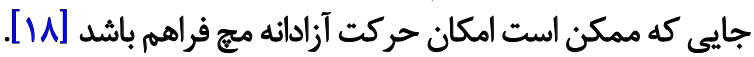

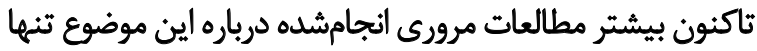

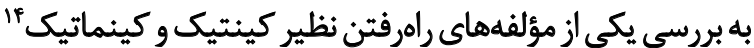

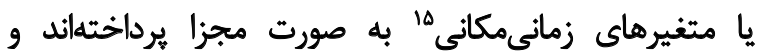

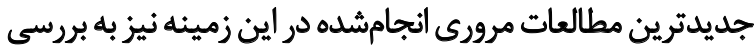

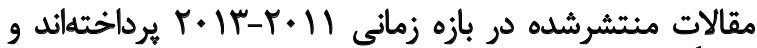

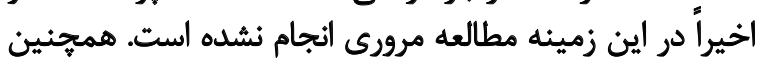

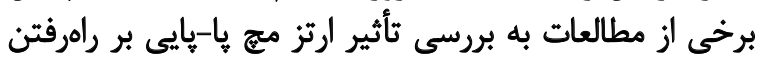

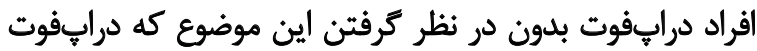

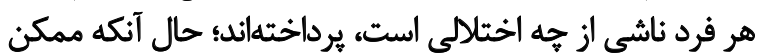

12. Clearance

13. Kinetic

14. Kinemtic

15. Spatio-temporal parameters

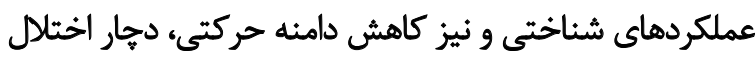

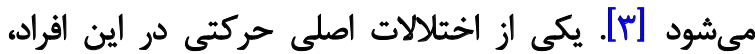

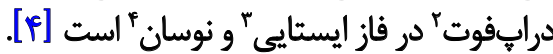

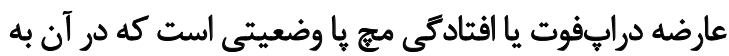

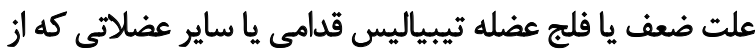

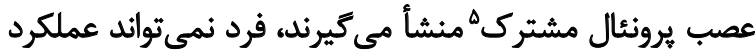

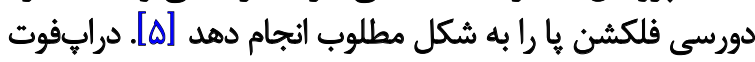

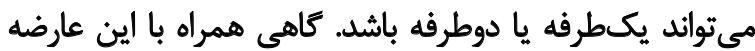

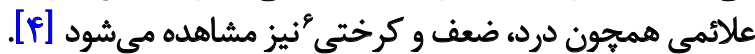

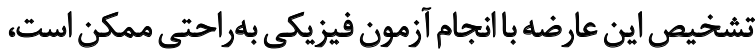

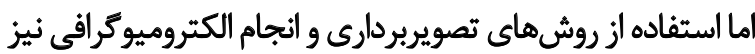
به بررسى دقيقتر اين عارضه كمك مى كند إعادئ.

افراد دجار دراضفوت زمان راهرفتن دجار مشكلاتى نظير

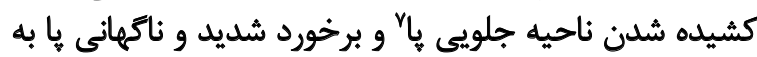

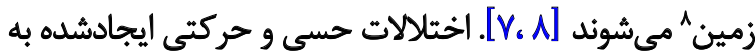

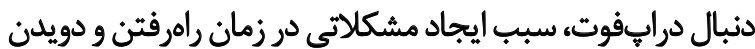

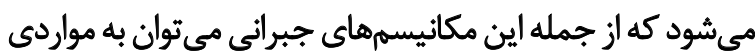

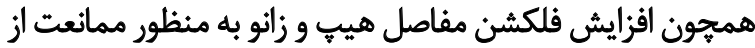

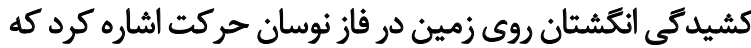

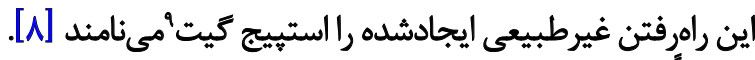

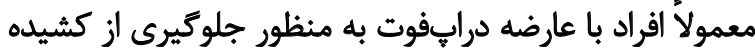

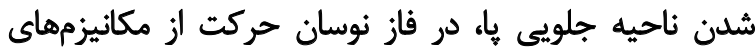

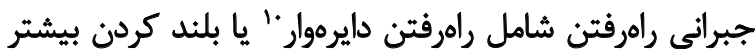

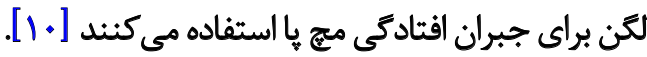

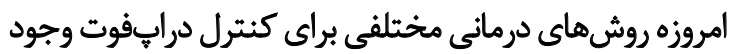

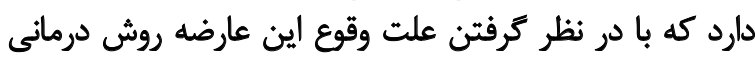

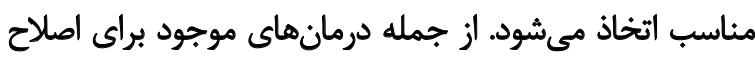

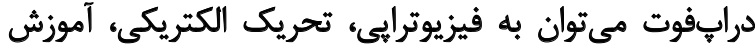

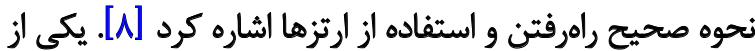

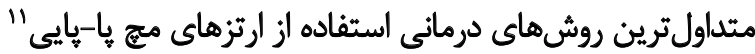

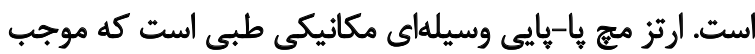

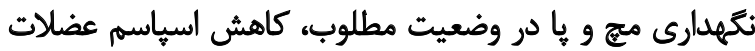

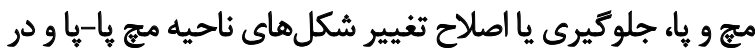

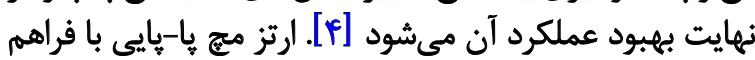

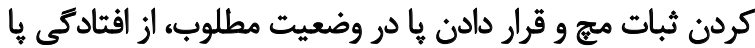

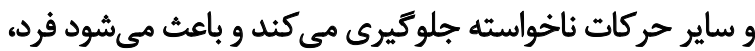

\section{Drop foot}

3. Stance phase

4. swing

5. Common Proneal

6. Numbness

7. Foot Drag

8. Foot slap

9. Steppage gait

10. Circumferential

11. Ankle Foot Orthoses (AFO) 


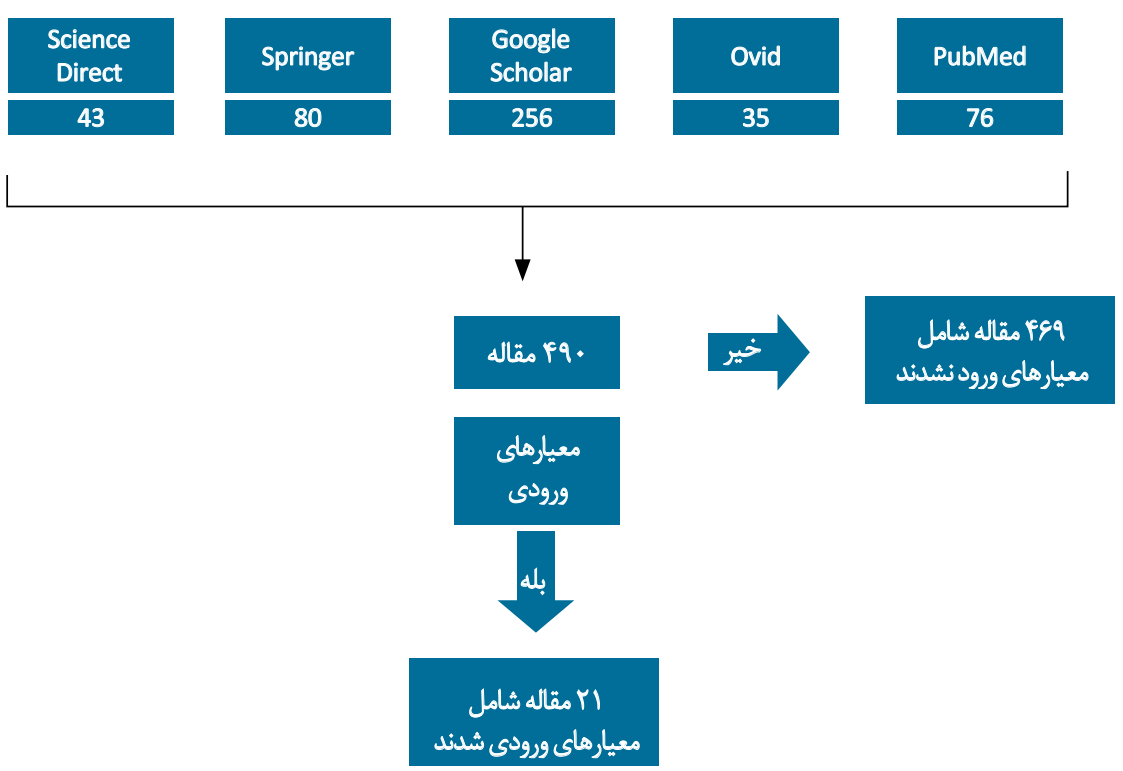

توانبخننى

راهرفتن، مؤلفههاي كينتيك و كينماتيك راهرفتن، سرعت ترادت

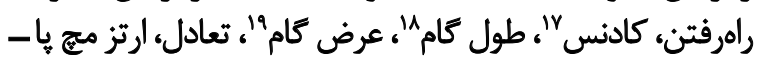

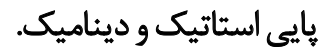

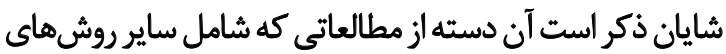

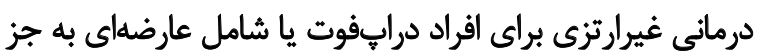

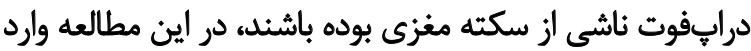

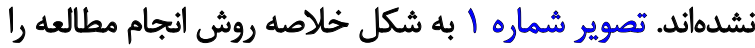
نشان مي دهد.

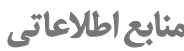
اين تحقيق بر اساس جستوجوى الكترونيكى در هايكاههاى

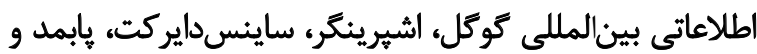

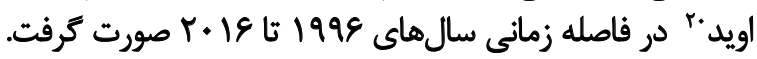

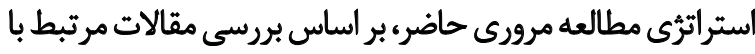

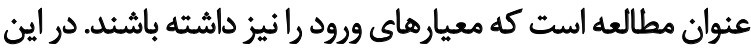

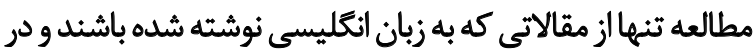
نشريات معتبر علمى به جاب رسيده باشنده استفاده شده است.

$$
\text { استراتثى جستوجو }
$$

مقاله حاضر شامل مطالعات انجامشده از سالهاي 1999 تأتان

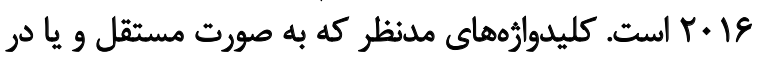

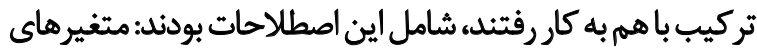

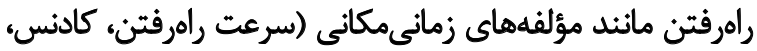

17. Cadence

18. Step length

19. Step width

20. ScienceDirect, Springer, Google Scholar, Pubmed, Ovid

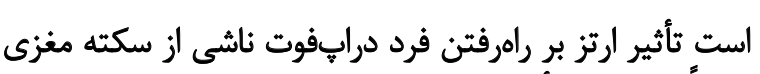

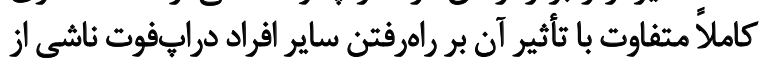

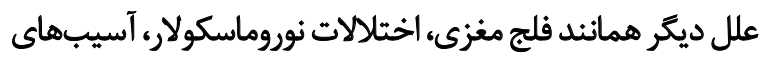
تروماتيك و غيره باشد. هدف از مطالعه حاضر، بررسى ثأثير ارتز بر تعادل و متغيرهاي

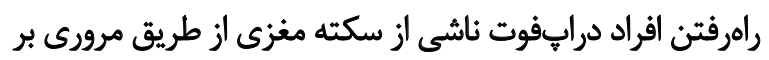

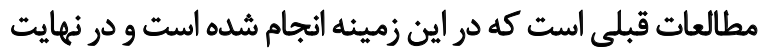

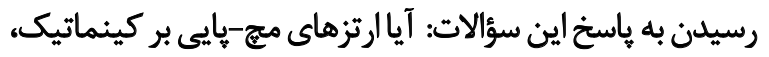

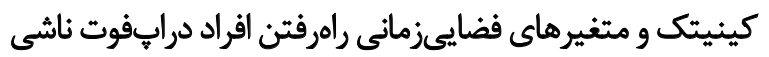

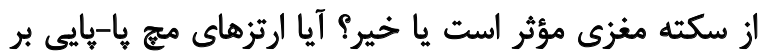

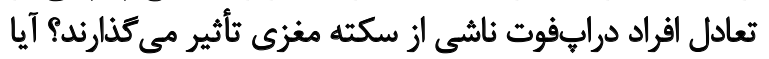

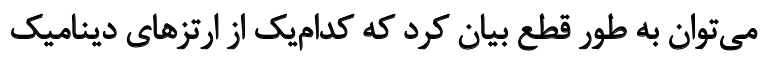

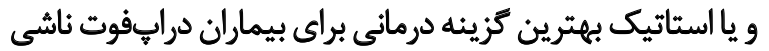

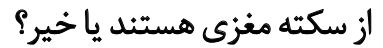

$$
\text { ووش بروسي }
$$

هعيار هاى واجد شُر إيط بودن برنامه

روش هاى استفادهشه براى انجام مطالعه مرورى حاضر براساس

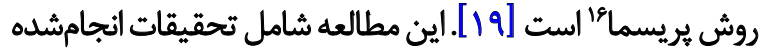

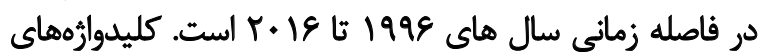

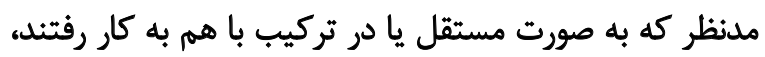

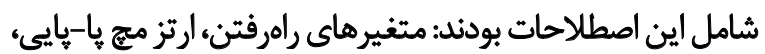

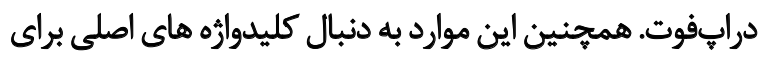

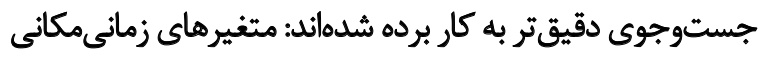

16. Preferred Reporting Item For Systematic Reviews And MetaAnalyses (PRISMA) 


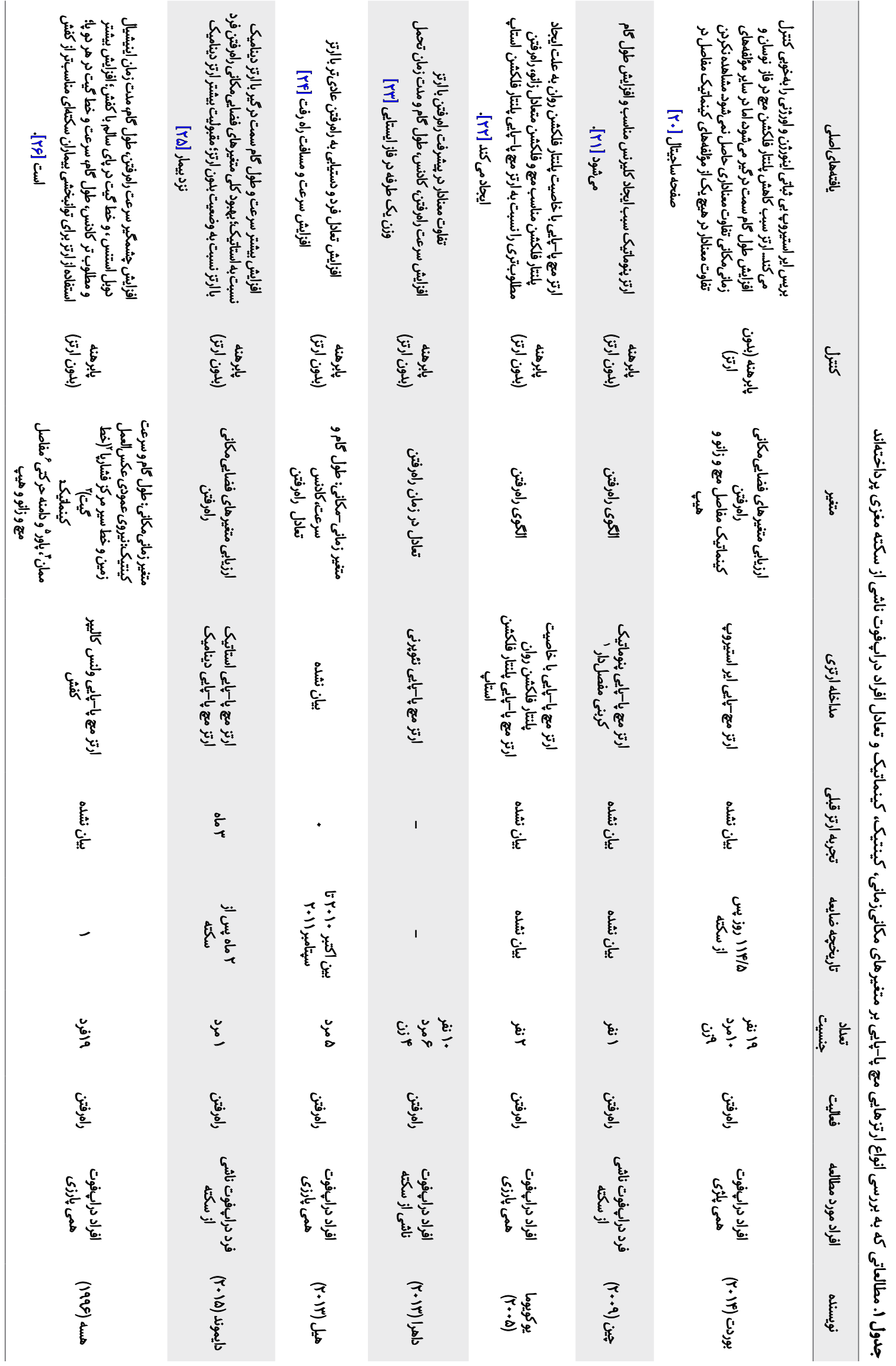




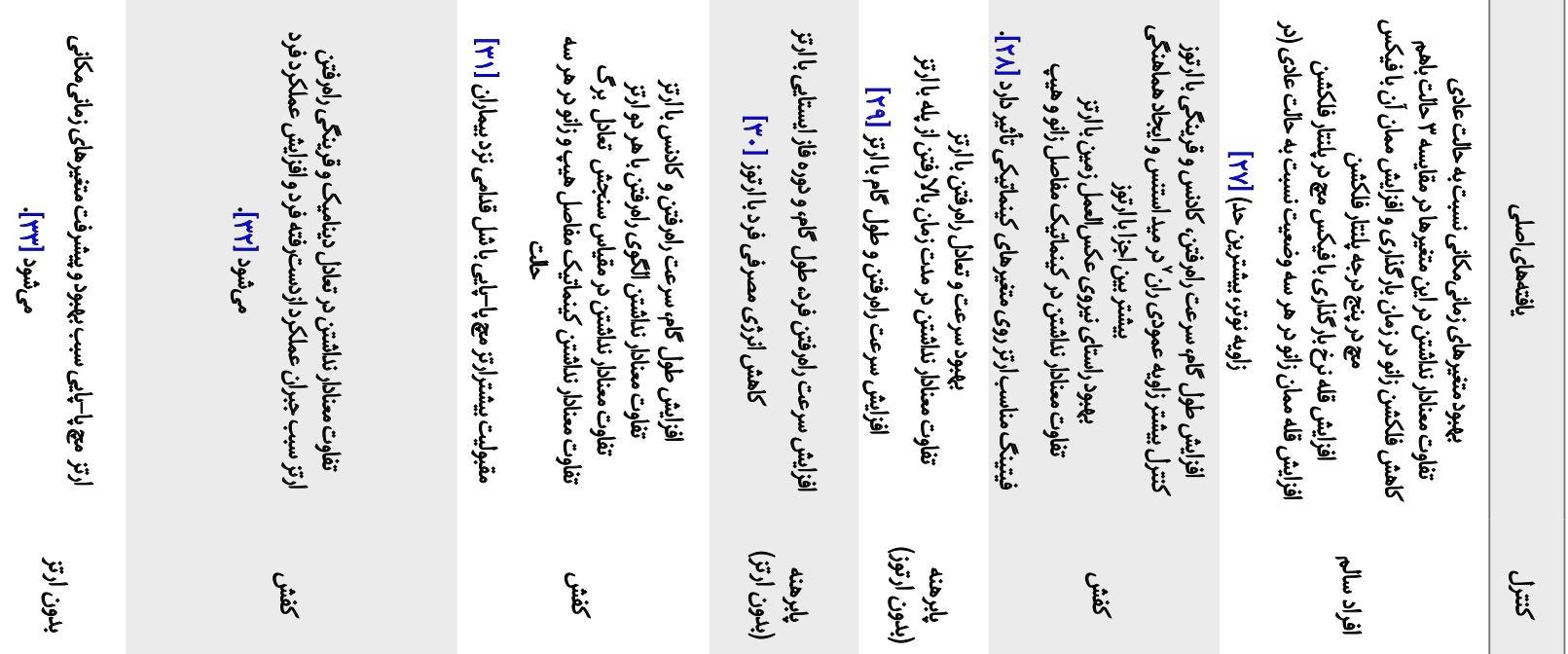

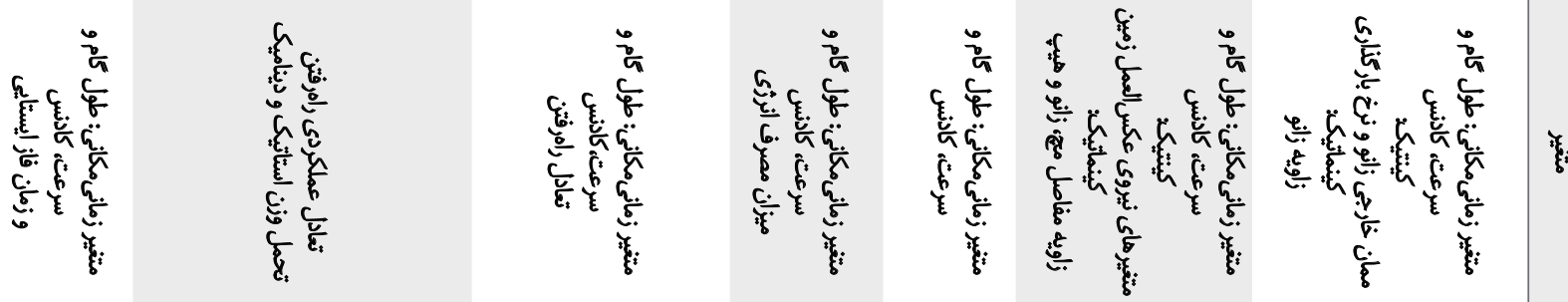

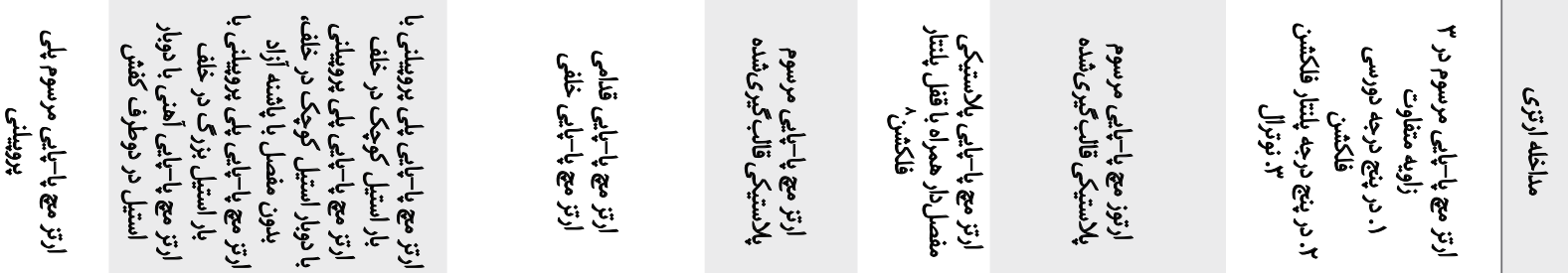

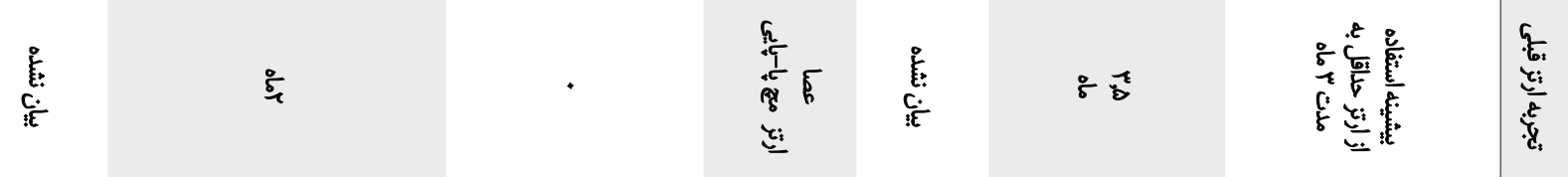

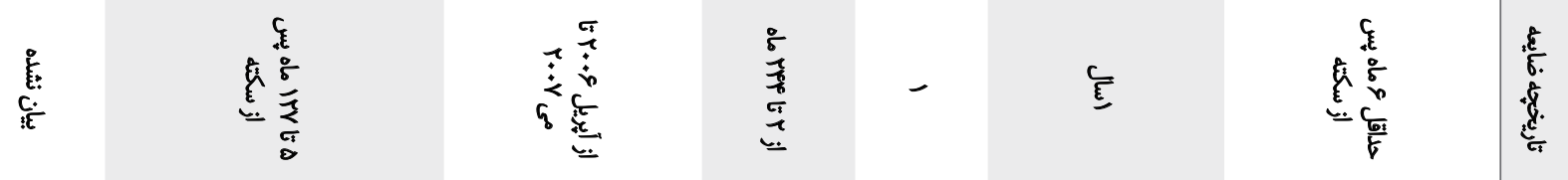

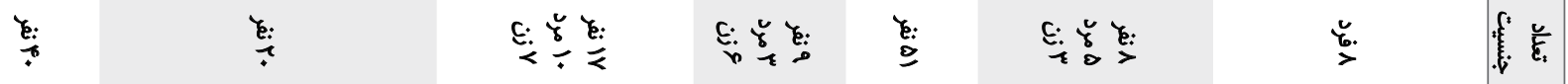

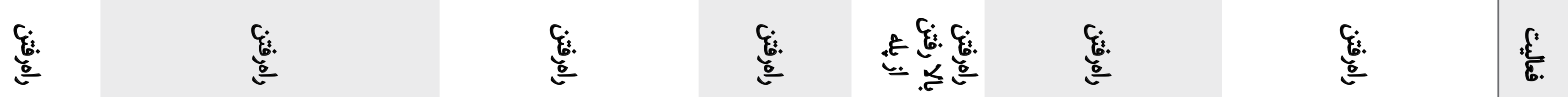

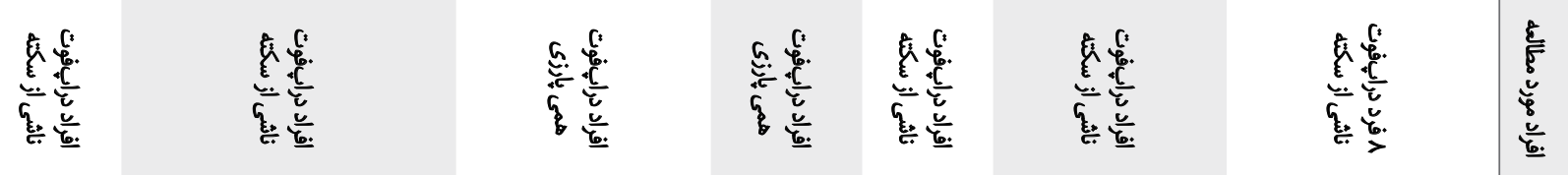

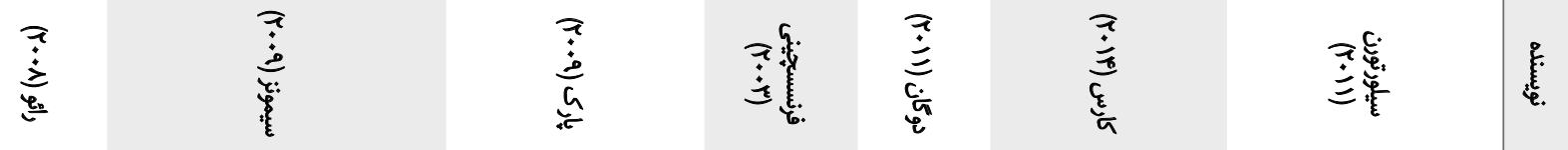



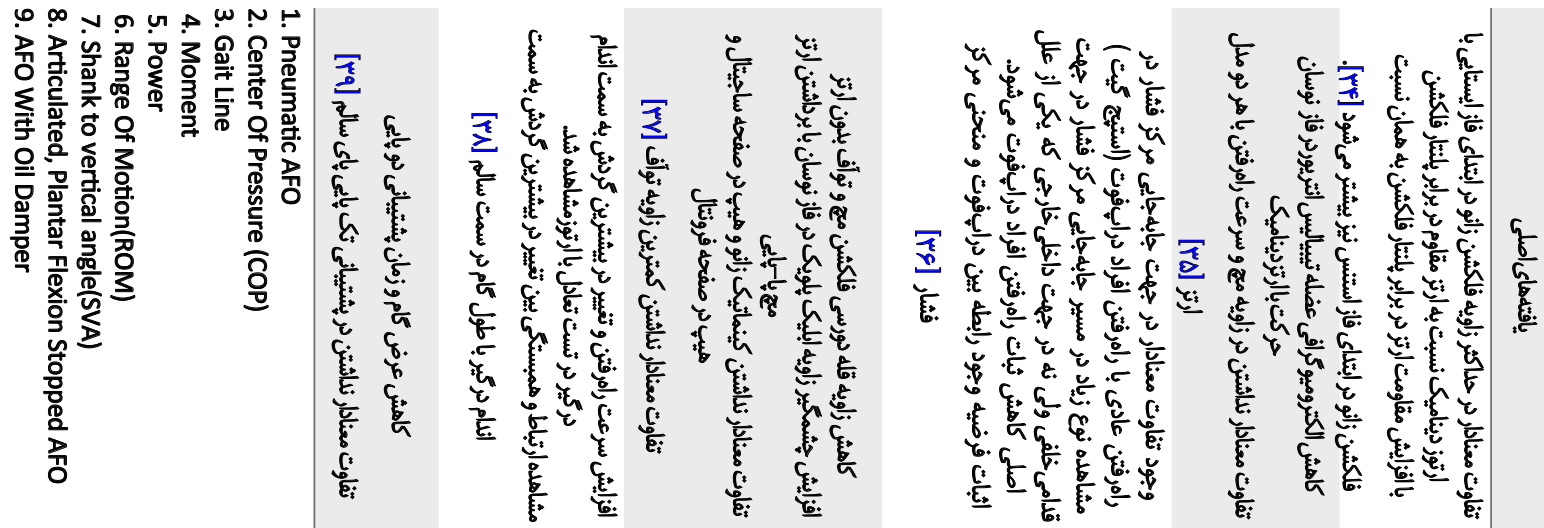

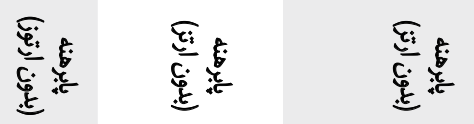

$\frac{2}{c}$

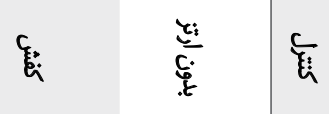

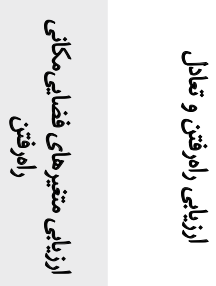

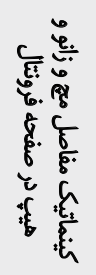

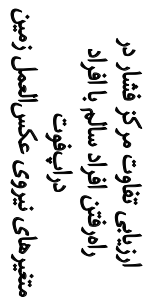

焉

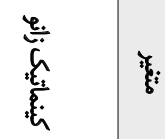

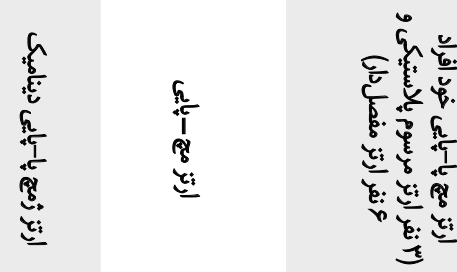

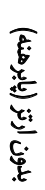

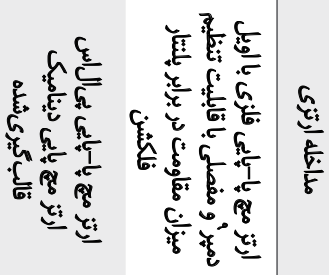

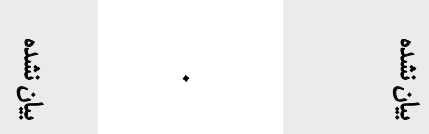

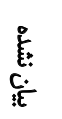

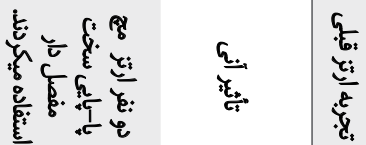

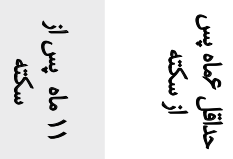

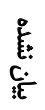

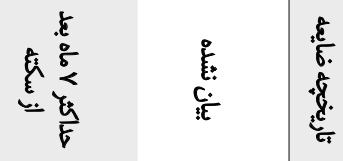

$\stackrel{\xi}{\xi}$

E.

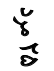

द्:

宕

鄢

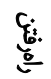

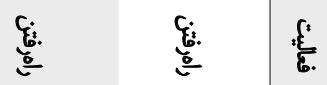

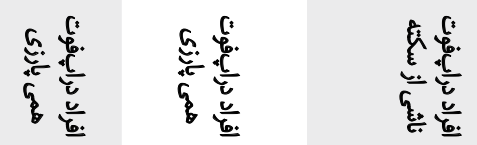

$\frac{c}{6}$

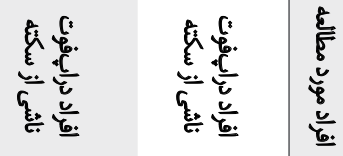

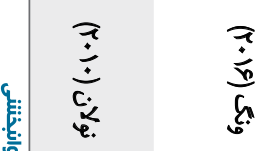

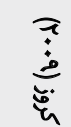

踩

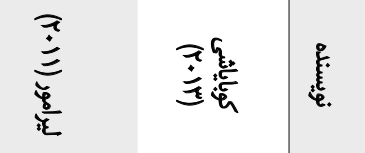


است. بعد از انتخاب نهايى موارد مناسب، متن كامل مقالات بر اساس

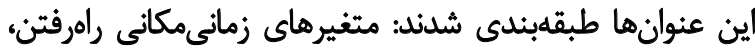

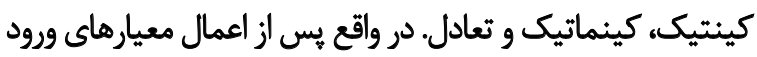

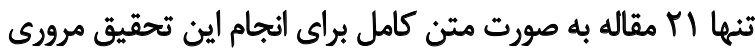

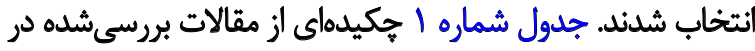
مطالعه حاضر و اطلاعات حاصل از آنها را انشان مى دهد.

\section{يافتهاهl}

اين تحقيق جكيدهاي از مطالعاتى است كه بر روى تأثير ارتز

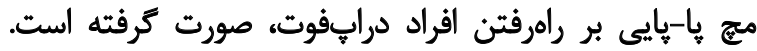

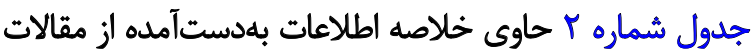

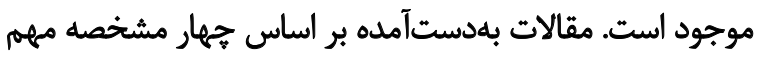

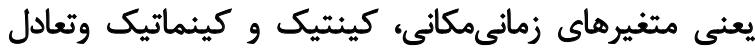

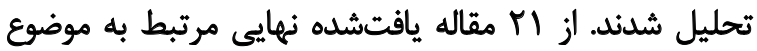

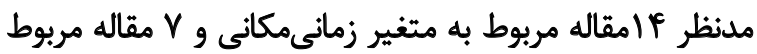

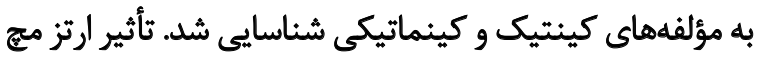

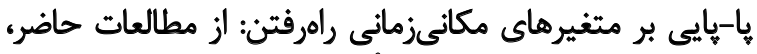

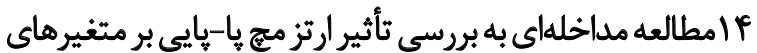
مكانى زمانى راهرفتن يرداختهاند.

$$
\text { سرعث راهرقثن }
$$

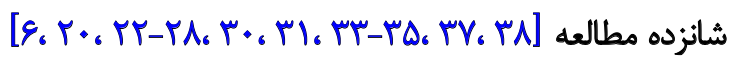

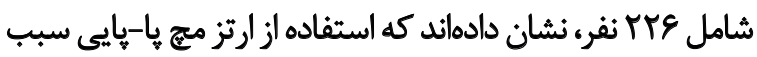

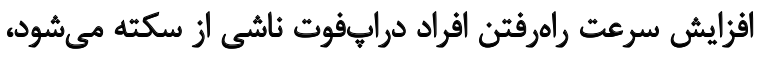

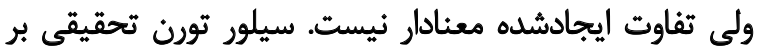

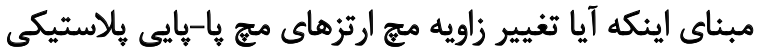

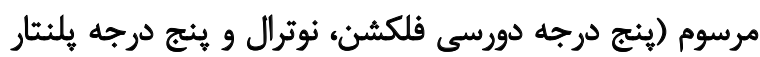

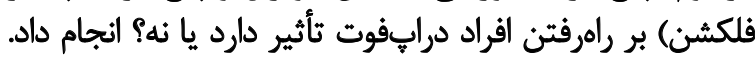

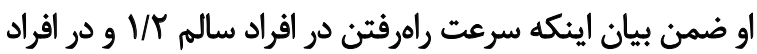

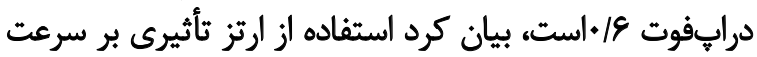

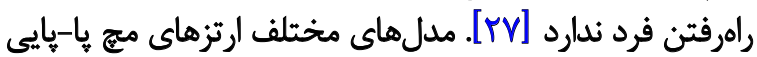

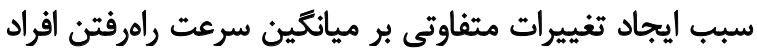

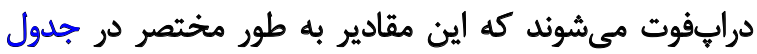
شماره Y ارائه شده است.

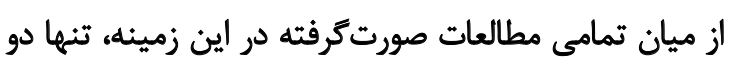

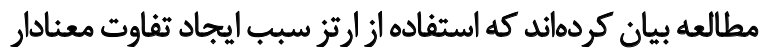

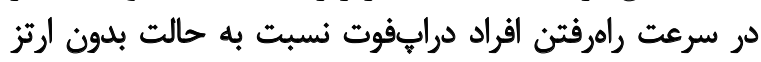

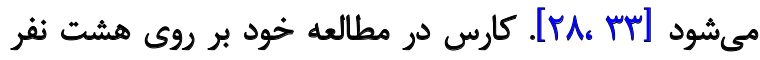

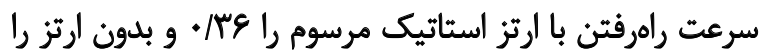

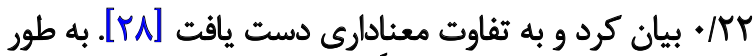

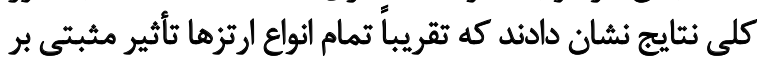
بهبود سرعت راهرفتن بيماران سكته مغزي دارند
طول و عرض كام)، كينتيك (نيروى عكس العمل زمين، مركز فشار

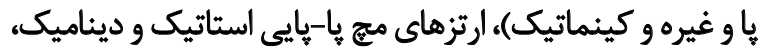

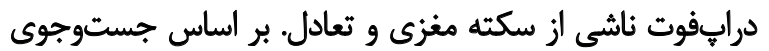

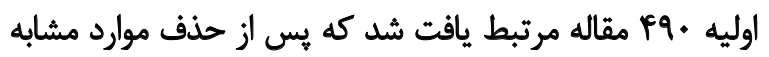

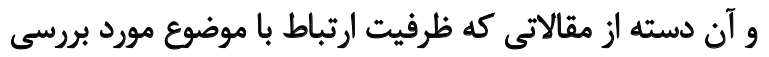

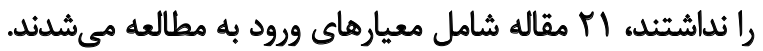

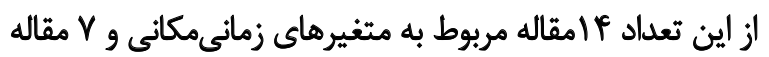

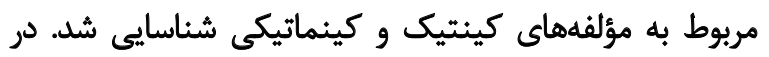

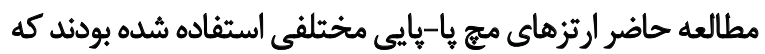

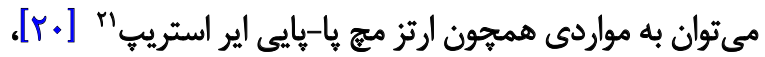

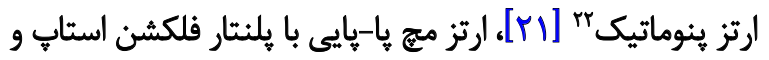

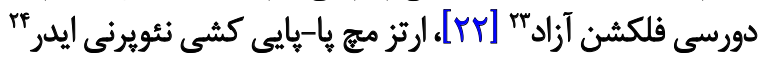

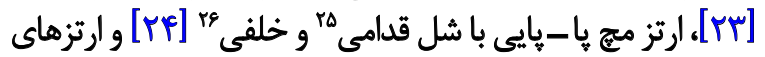

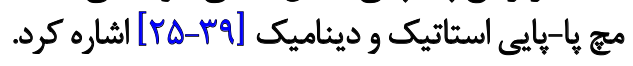

انتخاب مقالات

بعد از اتمام جستوجو، مقالات مرتبط به موضوع تحقيق بر ماند

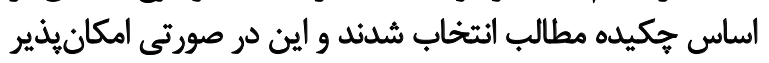

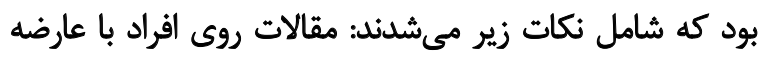

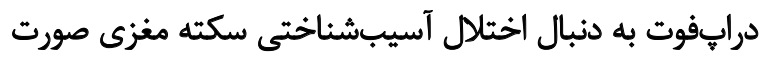

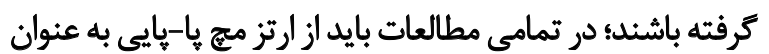

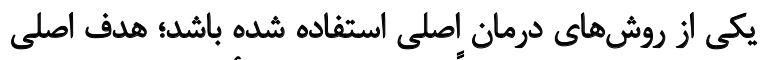

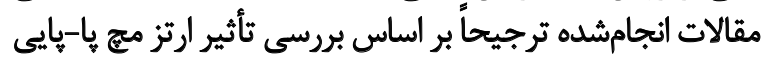

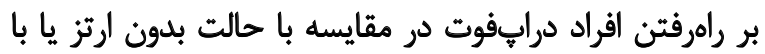

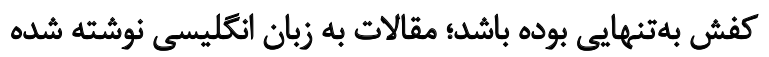

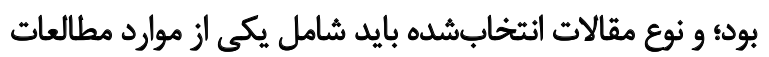

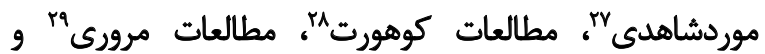

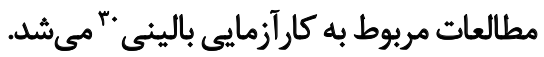

جمعآورى اطلاعاث

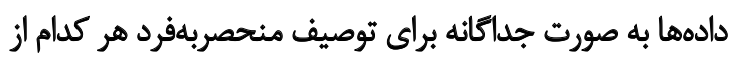

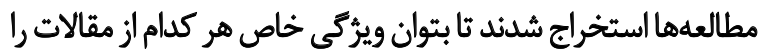

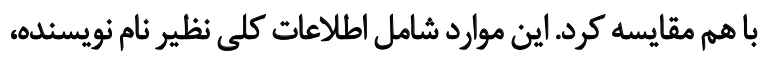

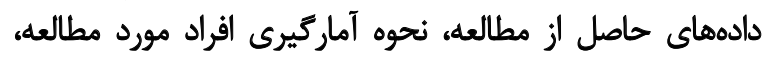

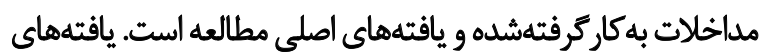
حاصل از اين مطالعه به صورت جكيدهاي ازئ ساير مطالعات بيان شده

\section{Air-stirrup AFO}

22. Pneumatic power harvesting ankle-foot orthosis

23. AFO with free dorsiflexion and plantar flexion stop

24. Elastic band orthosis (aider)

25. Anterior AFO

26. Posterior AFO

27. Single-subject studies

28. Cohort studies

29. Article reviews

30. Randomized control trial studies 
جدول r. مطالعاتى كه به بررسى تأثير انواع ارتزهاى مجع يا-باييى بر سرعت راهرفتن افراد درابفوت ناشى از سكته مغزى برداختهاند

\begin{tabular}{|c|c|c|c|c|c|c|c|c|c|}
\hline \multicolumn{7}{|c|}{ سرعت راهرفتن با مداخلات ارتزى } & \multirow[b]{2}{*}{ سبرعت رادرفتن } & \multirow[b]{2}{*}{ اقراد اد اد } & \multirow[b]{2}{*}{ 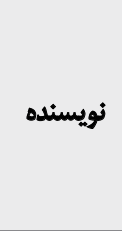 } \\
\hline ايراستيروب & 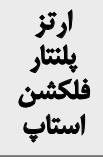 & فارتشيت & ارتز با شل & ارتز با شل & ارتز ديناميك & ارتز استاتيك & & & \\
\hline & & & & & $\cdot / \Delta T(\cdot / M)$ & & $\cdot \mid \Delta N(\cdot / \pi \cdot)$ & 1. & داهر \\
\hline & & & & & &.$/ \pi(-/ 1 \Delta)$ & $\cdot / T Y(\cdot / I V)$ & 19 & هبه \\
\hline & & & & & & $r V / r a(N / T+)$ & $10 / F V(F / 9 \Delta)$ & 9 & فرنسيسجينى \\
\hline & & & & & &.$/ \Delta r$ & & $\Delta$ & هيلر - l \\
\hline & & & $\Gamma T / Y \pm Y Y / q$ & $\Gamma T / q \pm T / T$ & & & $r e / 1 \pm r q$. & iv & 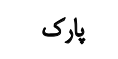 \\
\hline & & & & & & Eg/9P(TVVV) & ET/AT(YEMI) & $\Delta \wedge$ & ونك \\
\hline & $\cdot / A$ & $\cdot / A$ & & & & & & r & يوكويوما \\
\hline & & & & &.$/ 99$ &.$/ 91$ &.$/ A r$ & 1 & 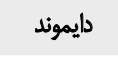 \\
\hline$g r / q(T / F)$ & & & & & & & $\Delta V / q(19)$ & 19 & 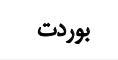 \\
\hline &.$/ \Delta r$ & .108 & & & & & & $ه$ & 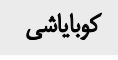 \\
\hline & & & & & $\cdot|\varphi q \pm \cdot / r|$ & $. / R T \pm . / . r \varphi$ & $. / 4 r \pm \cdot / / q$ & 10 & 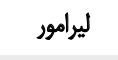 \\
\hline & & & & & &.$/ M T$ &.$/ 29$ & 9 & 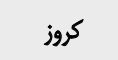 \\
\hline
\end{tabular}

Ff ID كادثن

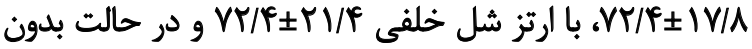
ارتز

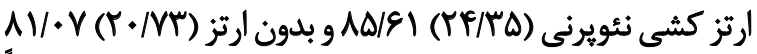

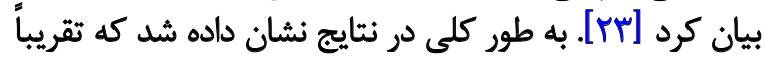

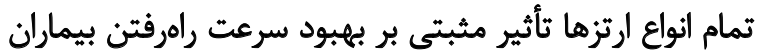
سكته مغزى دارند، اما معنادار نيست.

\section{طول كام سمت مبثلا}

سيلور تورن در مطالعه خود طول كام سمت مبتلا در افراد

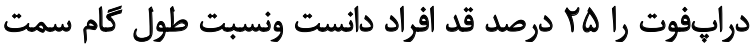

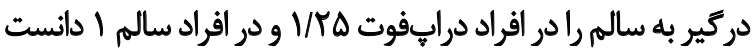

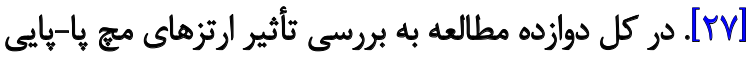

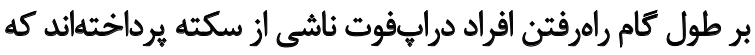

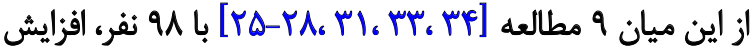

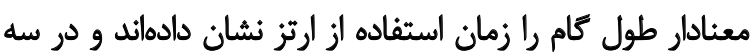

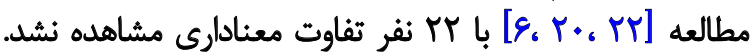

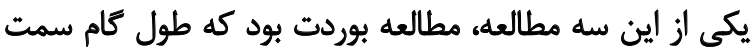

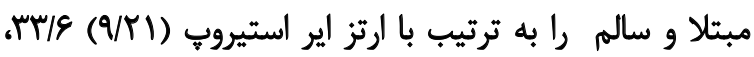
(If/T)

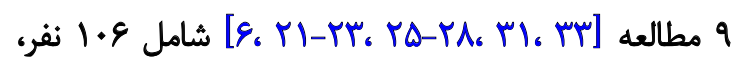

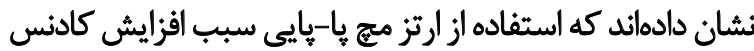

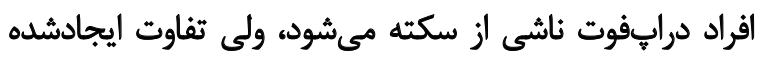

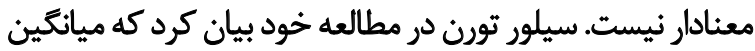

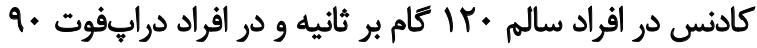

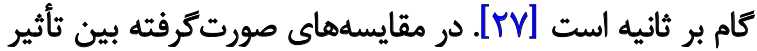

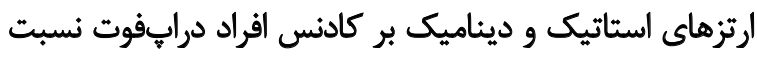

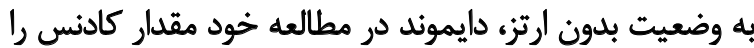

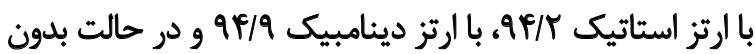

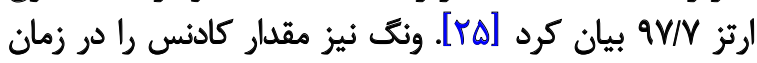

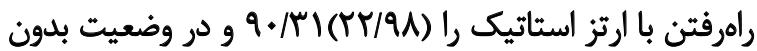

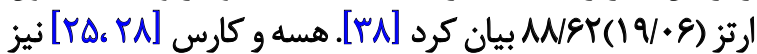

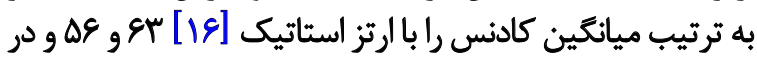

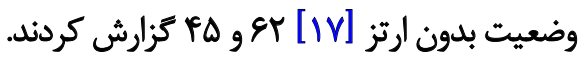

از ميان ساير مطالعاتى كه به بررسى برخى مدلهاي ديخًر

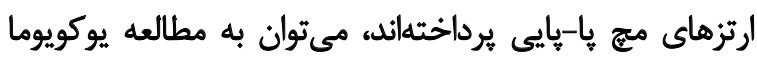

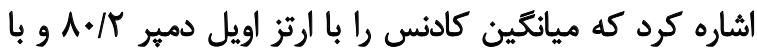

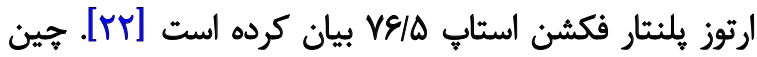

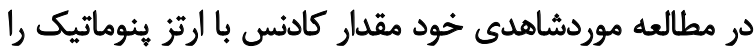




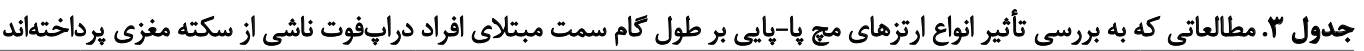

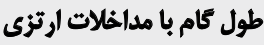

\begin{tabular}{|c|c|c|c|c|c|c|c|c|c|}
\hline ايراستيروب ارتز & أرلز استينتار & فلكشن رواصيت & ارتز با شلى & ارتزامى شل & ارتز ديناميك & ارتز استاتيك & طول كام (بدون & افراد & ويسنده \\
\hline & & & & & PE/FI (NTA) & & TE/OP (NTE) & 1. & sols \\
\hline & & & & & & $\cdot / 8 \Delta(\cdot / M A)$ & $\cdot / \& Y(\cdot / 1 V)$ & 19 & هسه \\
\hline & & & & & &.$/ T 1$ &.$/ 29$ & 9 & كروز \\
\hline & & & SV/ $\Delta \pm 19 / 9$ & $E / / 8 \pm 19 / q$ & & & $\Delta \varepsilon / F \pm M / V$ & IV & هارك \\
\hline & & & & & & $P f / \Delta M(I T / / 9)$ & $T T / T q(T / T V)$ & $\Delta \mathcal{M}$ & وئك \\
\hline & & & & & Tal. & .101 &.$|\Delta|$ & 1 & دايموند \\
\hline
\end{tabular}

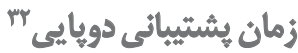

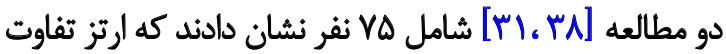

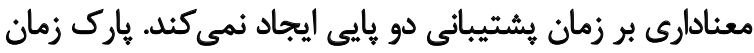

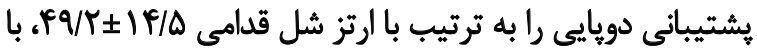

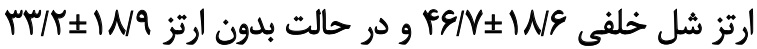

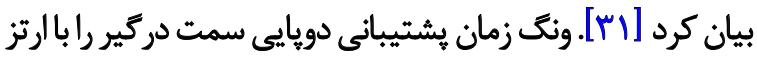

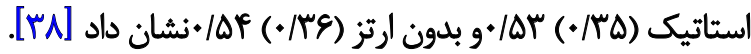

$$
\text { زمان }
$$

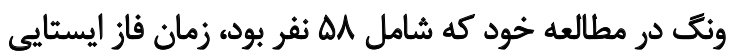

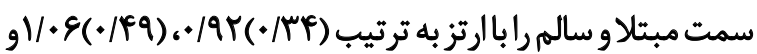

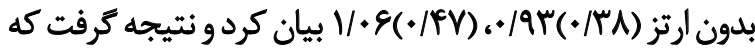

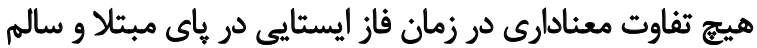

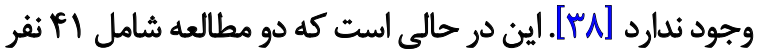

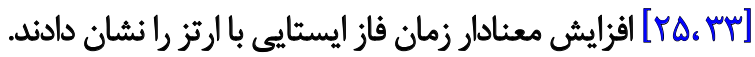

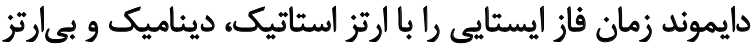

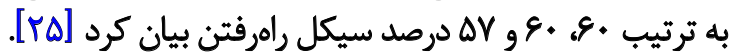

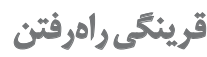

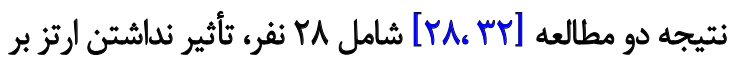

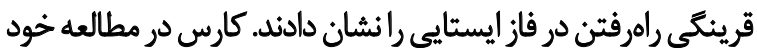

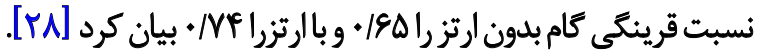

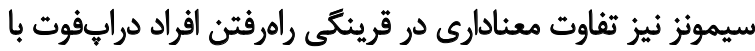

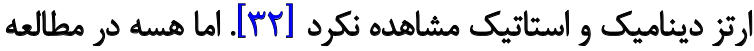

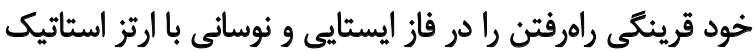

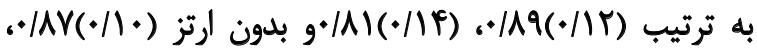

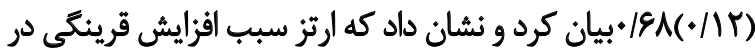

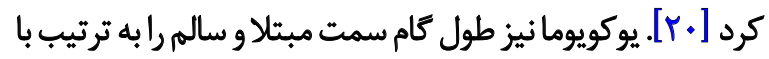

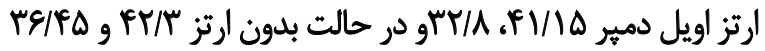

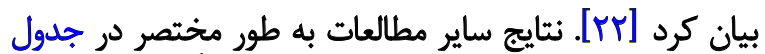

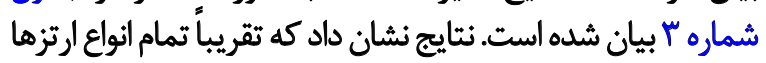
تأثير مثبتى بر بهيبود طول كام بيماران سكته نئه مغزى دارند.

$$
\text { عرض كام }
$$

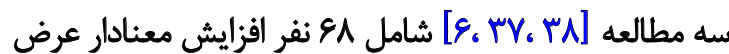

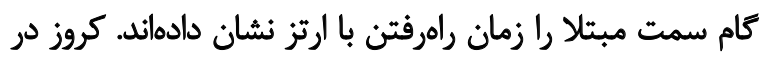

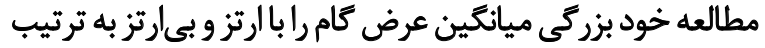

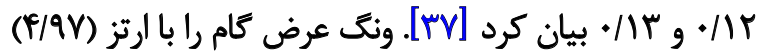

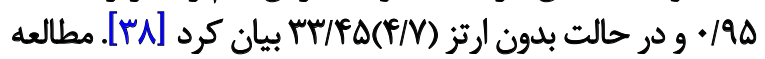

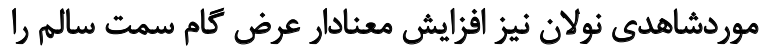

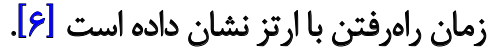

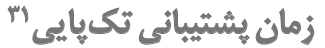

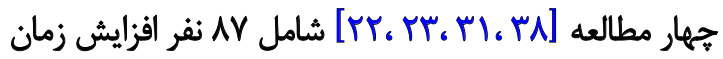

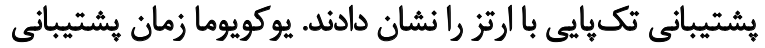

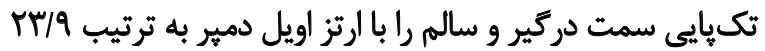

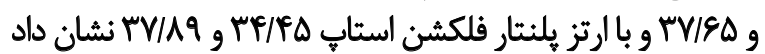

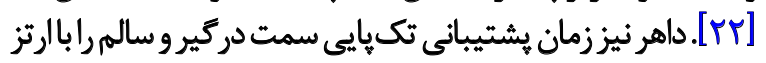

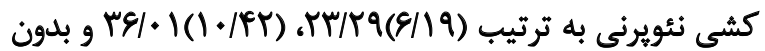

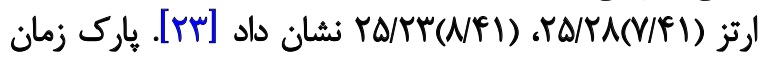

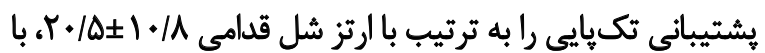
ارتز شل خلفى

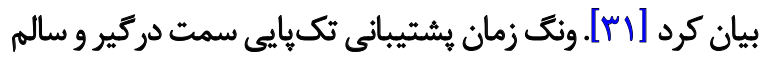

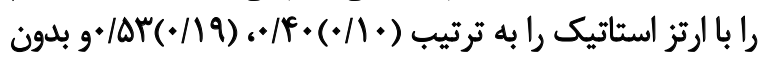
ارتز (1/1) 
كاهش نرخ باركذارى سمت سالم و تغييرنكردن نرخ باركذارى

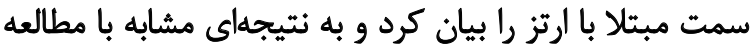

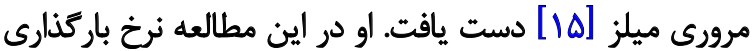

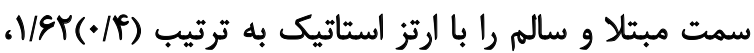

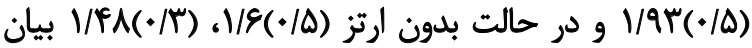

كرد [rs

\section{كينماتيكميج}

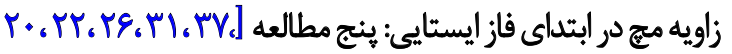

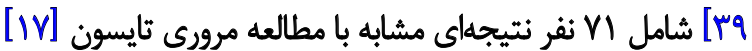

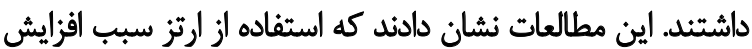
معنادار زاويه ميج در شروع فاز ايستايى مى ائشود.

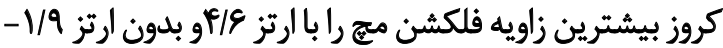

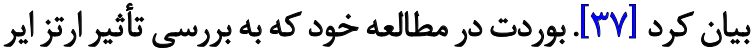

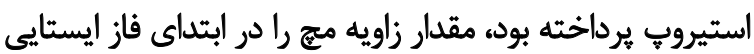

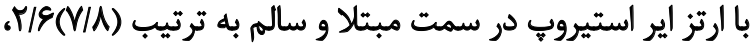

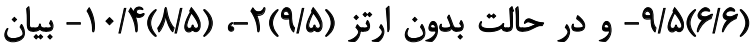

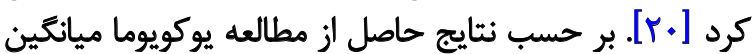

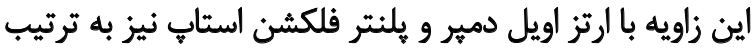

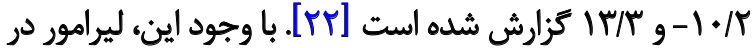

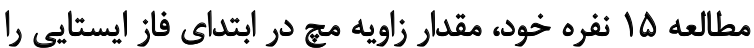

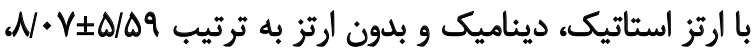

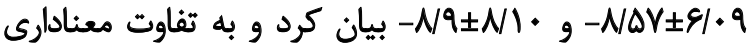

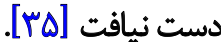

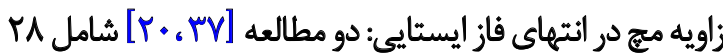

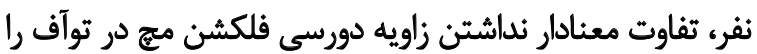

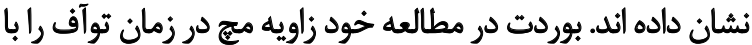

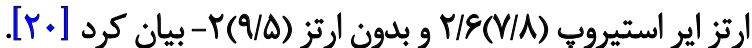

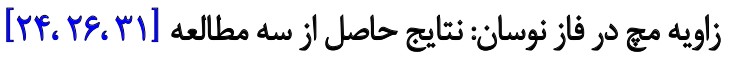

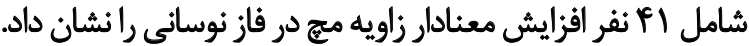

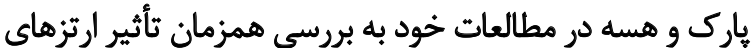

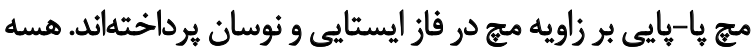

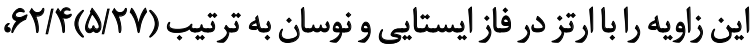

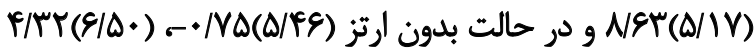

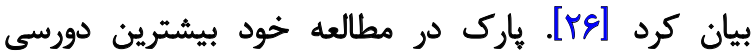

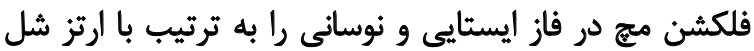
قدامى (I/T/F)

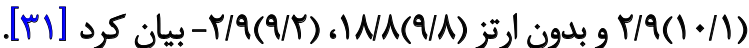

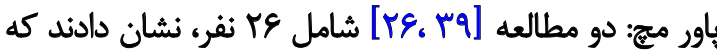

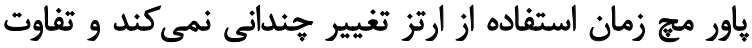

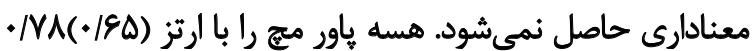

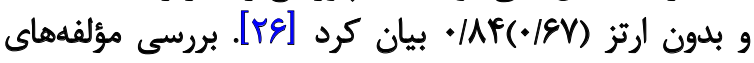

فاز نوسانى راهرفتن افراد درابفوت مىشود [عץ]].

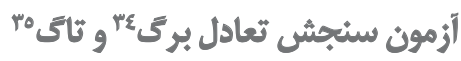

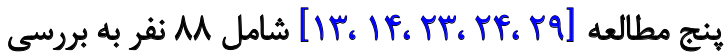

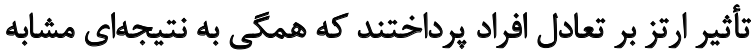

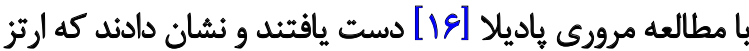

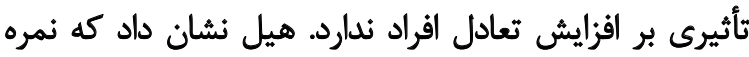

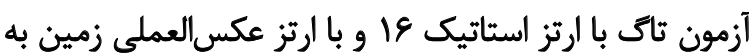

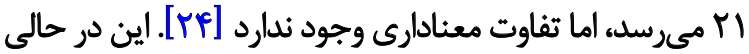

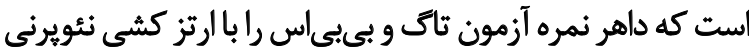

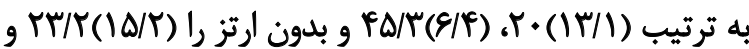
Fr/V(V/I)

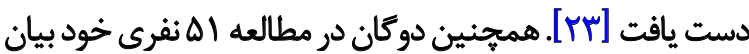

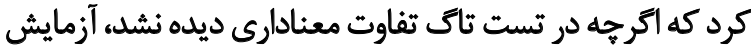

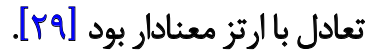

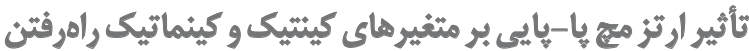

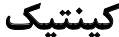

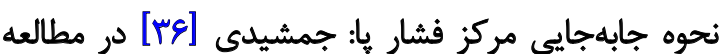

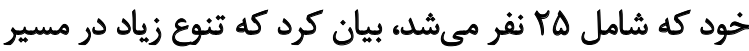

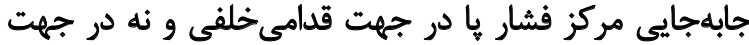

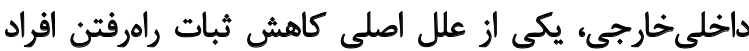

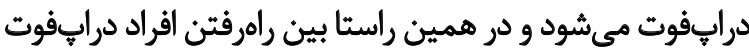

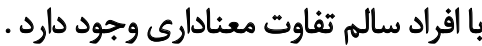

بيشترين نيرو ضربهاى باشنه زمان باركذارى بأ: سيلور تورن

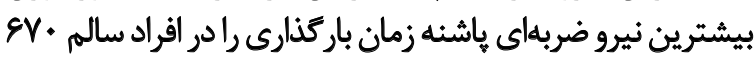

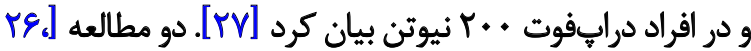

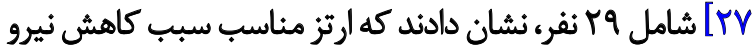

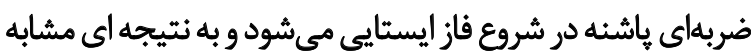

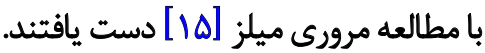

نيروى عمودى عكسالعمل زمين: هسه در مطالعه خود برديه

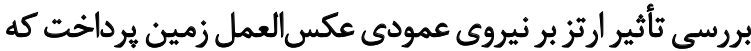

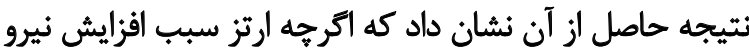

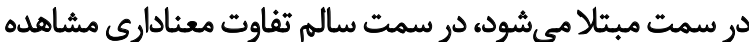

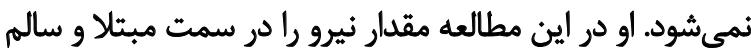

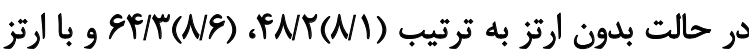

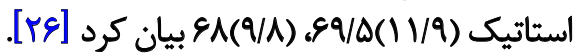
نرخ بار تذارى "iv هسه در مطالعه خود كه شامل آ نفر مىشده

34. The Berg Balance Scale(BBS)

35. Time Up\& Go Test(TUG)

36. Maximum of Impact Force in Loading Responce

37. Loading Rate 


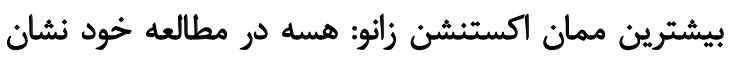

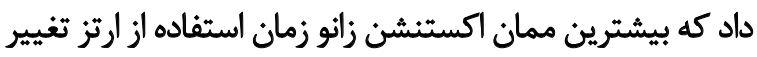

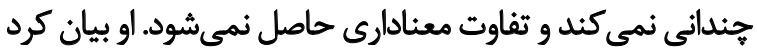

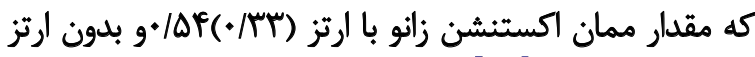

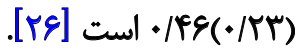
كينماتيك هيب بيشترين فلكشن هيب و اكستنشن در ابتداي فاز ايستايي:

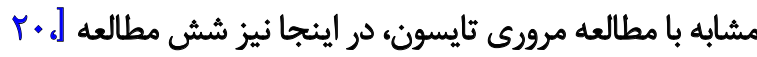

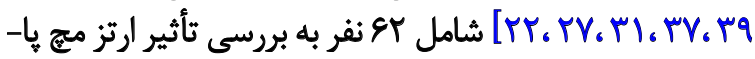

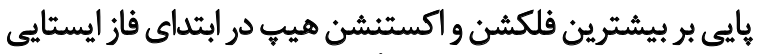

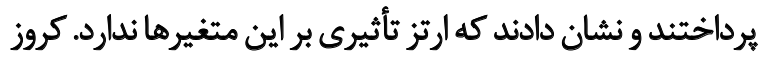

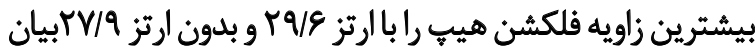

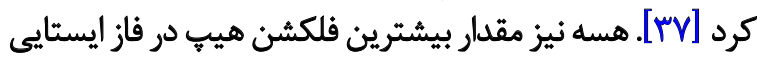

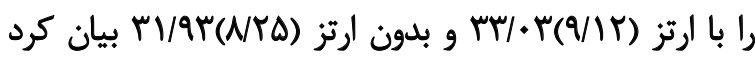

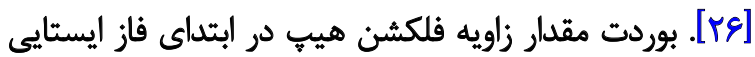

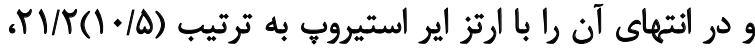

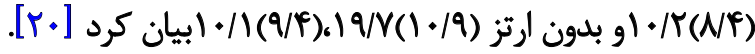

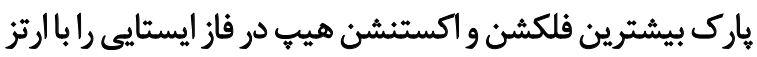

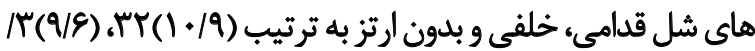

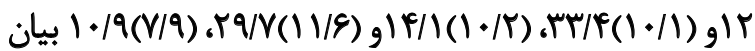

كرد [1)

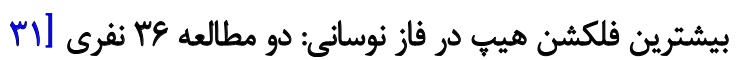

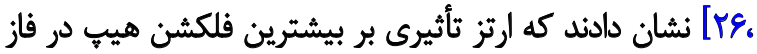

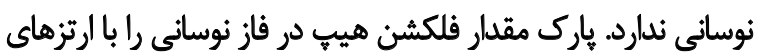

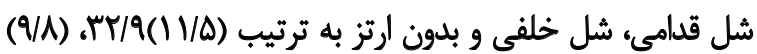

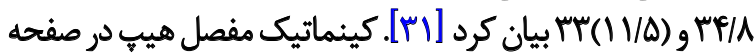

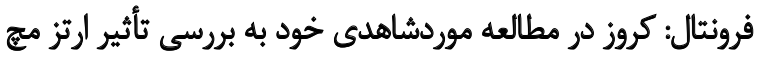

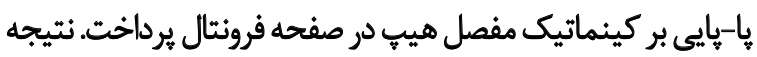

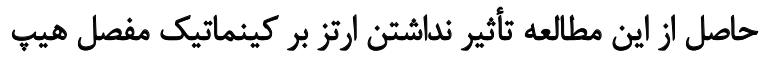

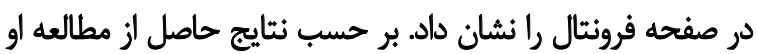

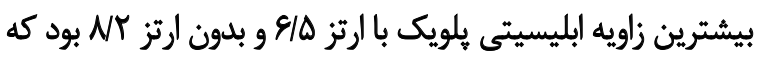

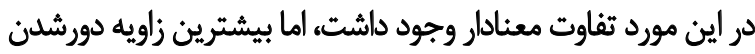

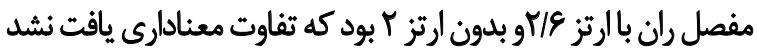

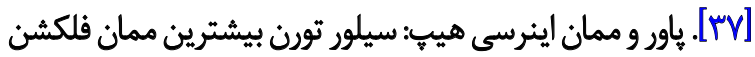

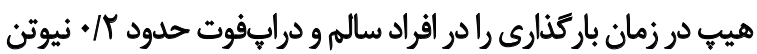

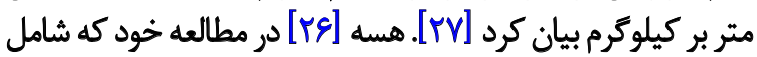

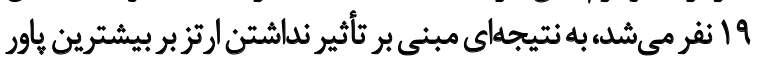
مفصل هيب در فاز نوسانى دست يافت.

ثک

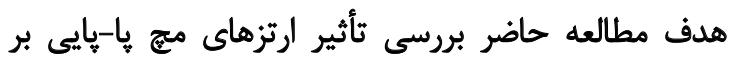

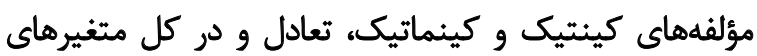

كينتيكى حاصل مطالعه بركمن نشان داد كه استفاده از ارتز

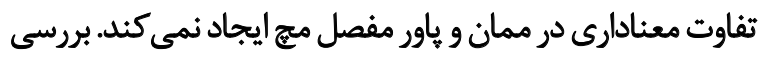

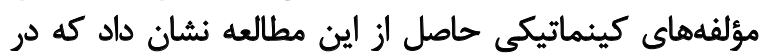

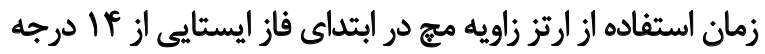

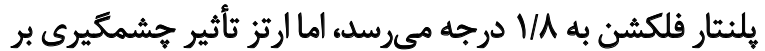

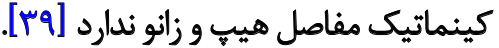

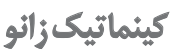

بيشترين فلكشن زانو در فاز ايستايى: سيلور تورن مقدار

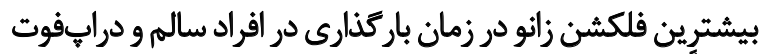

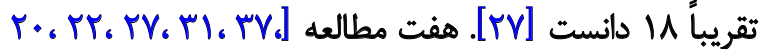

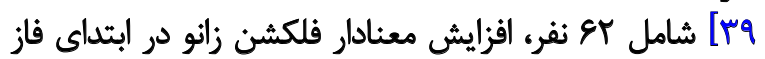

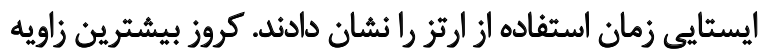

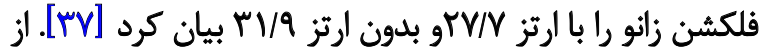

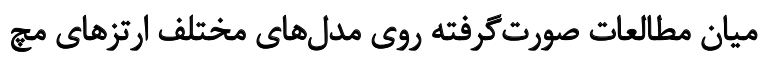

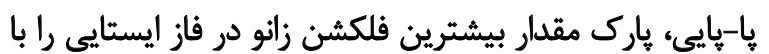

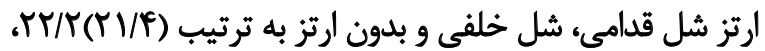

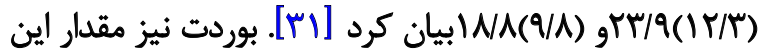

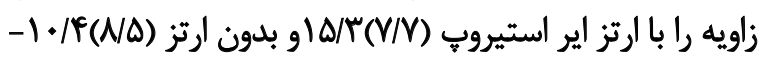

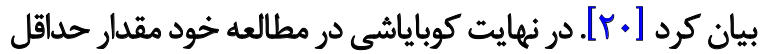

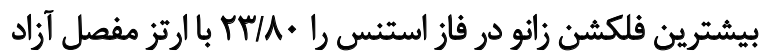

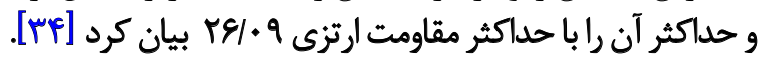

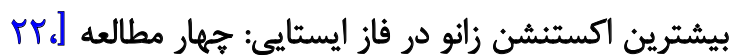

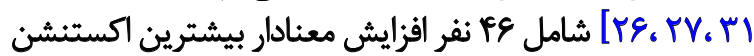

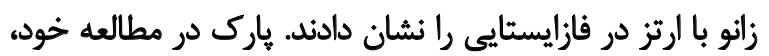

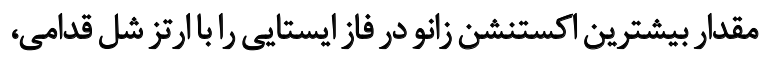

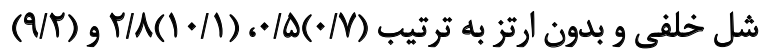

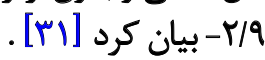

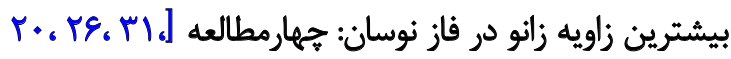

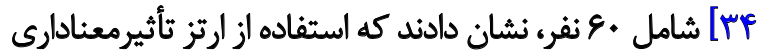

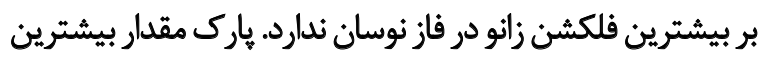

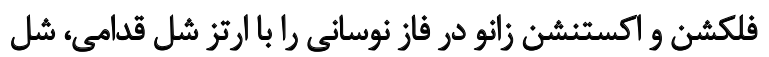

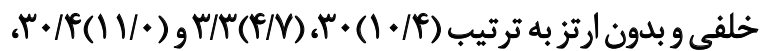
( فلكش

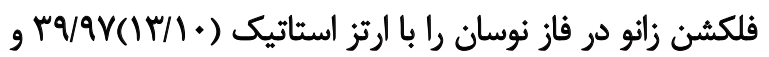

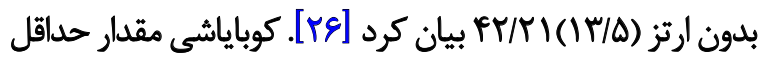

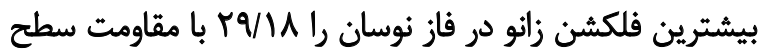

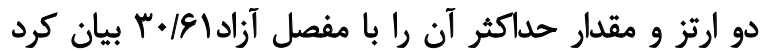

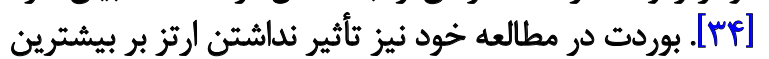

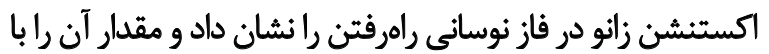

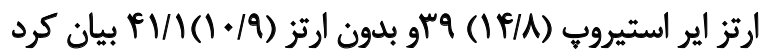

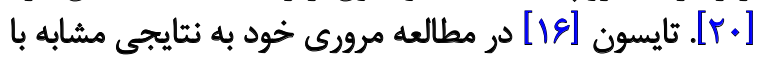
مطالعات مذكور دست يافته بود. 
مطالعات انجامشده به نظر مىرسد كه از بين تمامى مدل هاي

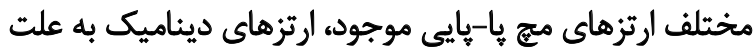

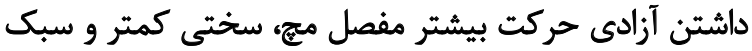

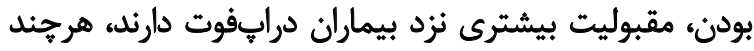

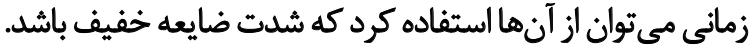

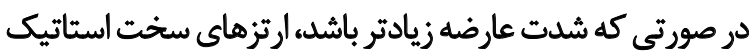

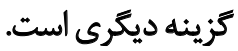

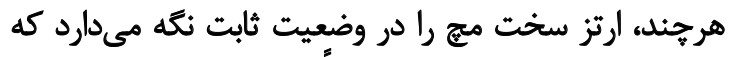

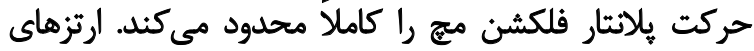

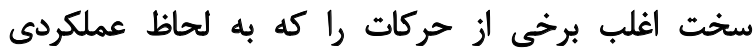

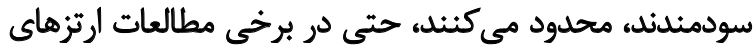

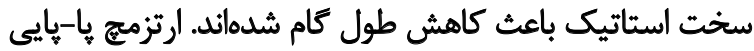

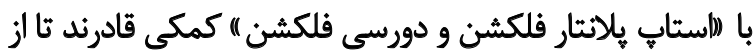

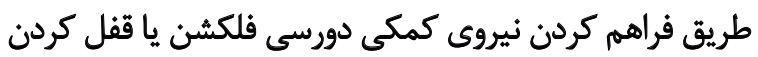

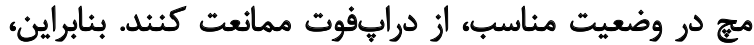

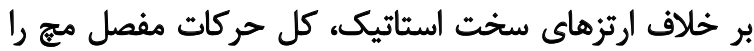

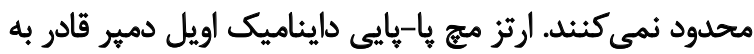

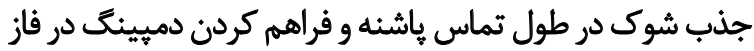

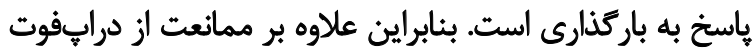

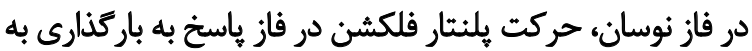

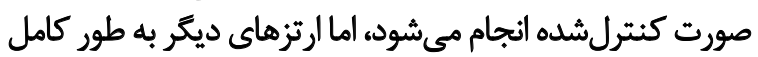
از حركت يُلنتار فلكشن در اين فاز ممانعت مى كرديند.

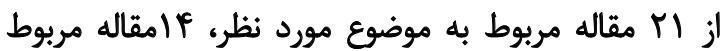

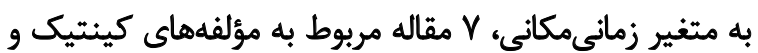

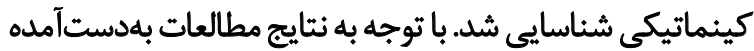

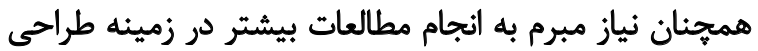

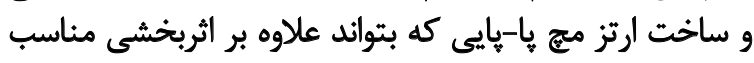

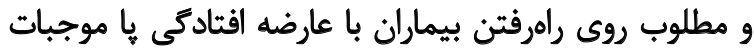

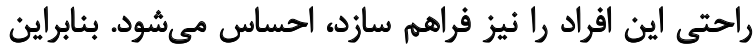

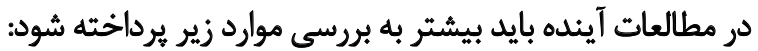

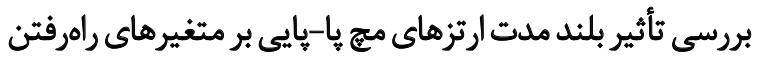

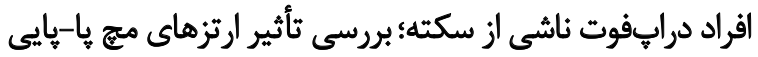

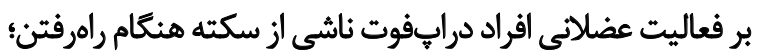

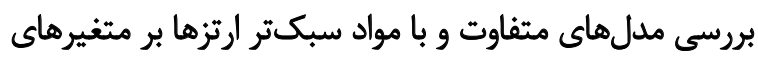

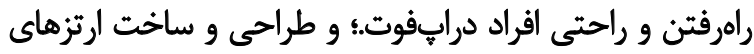

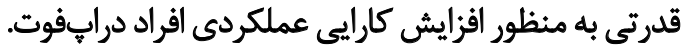

\section{نتيجلهيّيرى}

با توجه به نتايج جمعبندى شده از مطالعات انجام شده، تمامى مأى

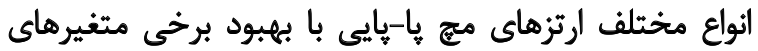

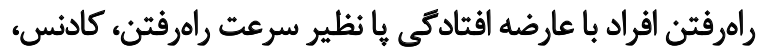

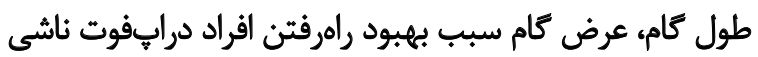

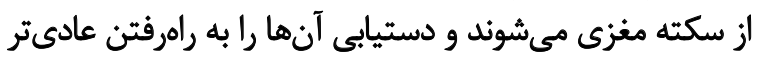

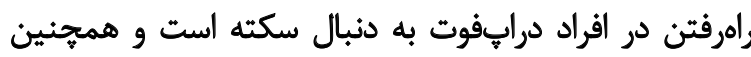

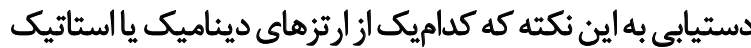

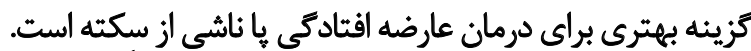

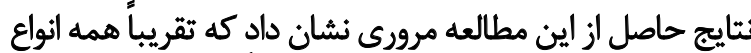

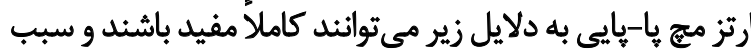

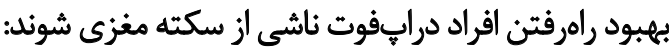

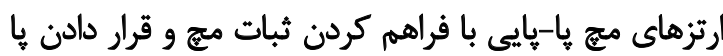

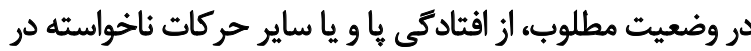

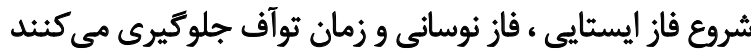

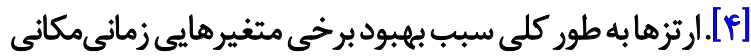

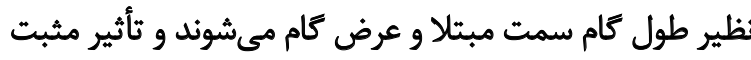

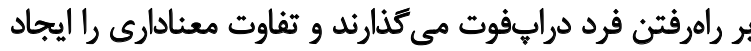

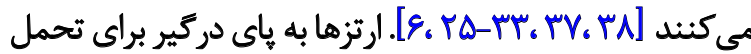

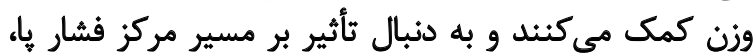

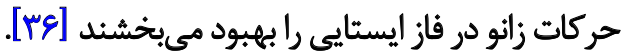

ارتزهاى ميج - يايیى سبب بهبود مؤلفههاى كينتيكى راهرفنن

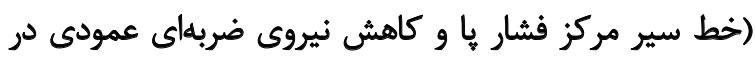

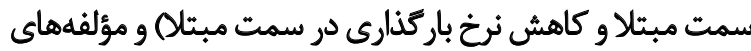

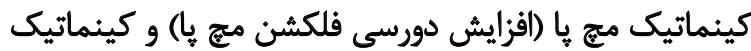

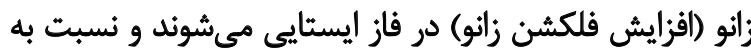

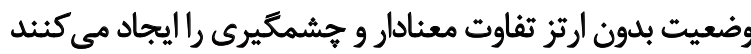

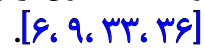

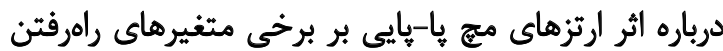

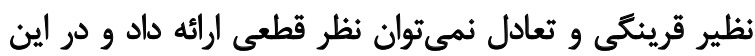

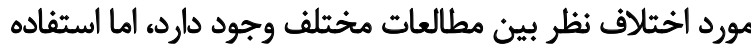

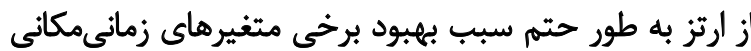

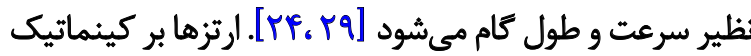

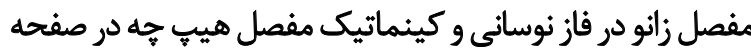

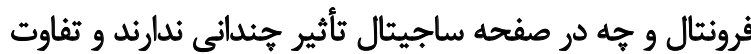
معنادارى ايجاد نمى كنيند [rV]

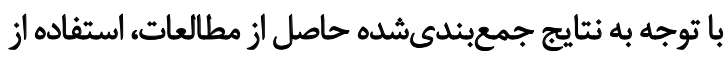

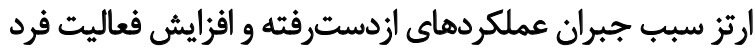

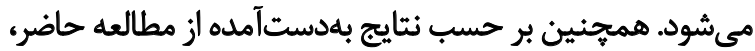

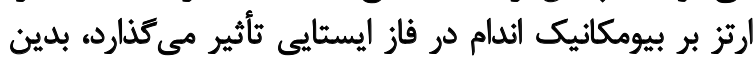

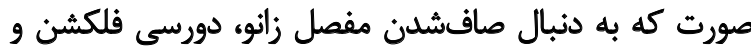

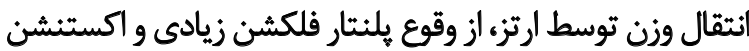

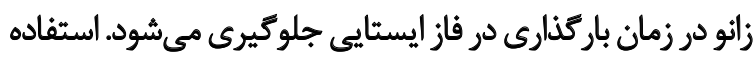

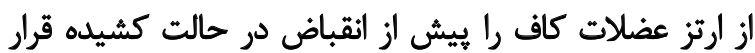

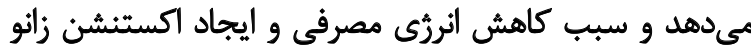

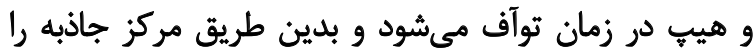

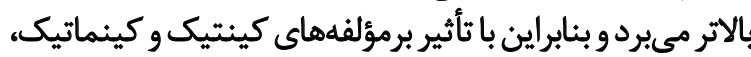

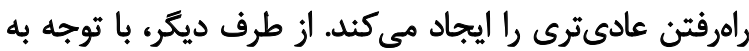


نسبت به وضعيت بدون ارتز امكانيذير مي كنثد. به نظر مىرسد

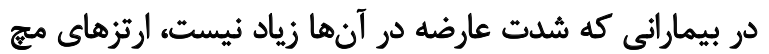

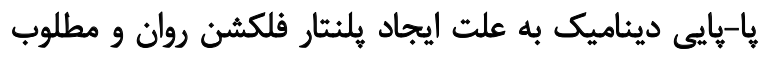

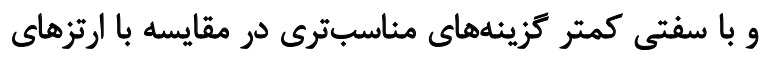

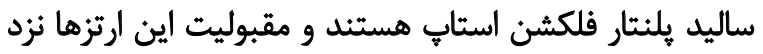

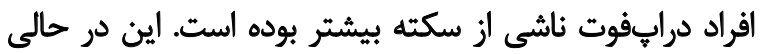

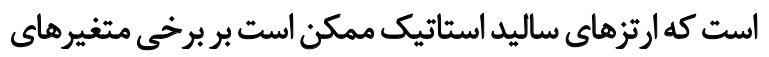

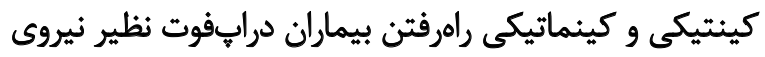

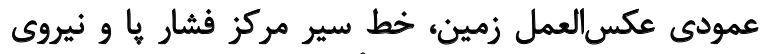

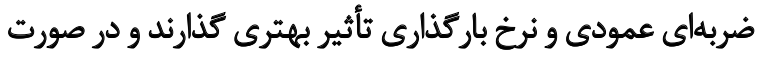

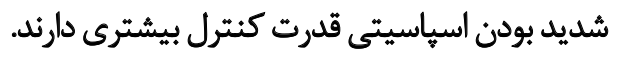

بنابراين با توجه به اين نكته كه ارتزهاى مج يا-ياييى با طرح

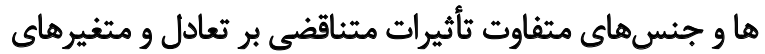

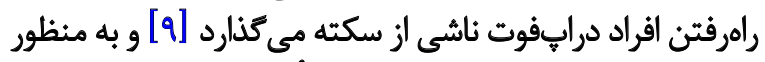

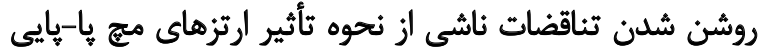

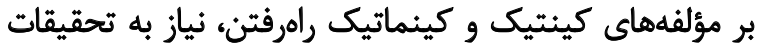

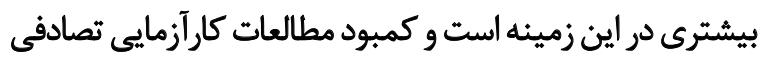

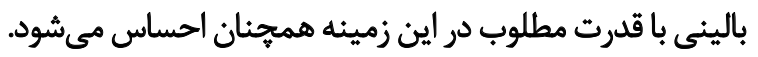

$$
\text { تشكر و قمدرهافي }
$$

اين مقاله حامى مالى نداشته است. 


\section{References}

[1] Shamsedini AR, Holisaz MT, Keyhani MR. [Comparison of balance abilities of patients with right and left hemiplegics stroke (Persian)]. Archives of Rehabilitation. 2008; 8(4):35-8.

[2] Hassan Abadi M, Hajiaghaee B, Saeedi H, Amini N. [The immediate effect of a textured insole in nonparetic lower limb symmetry of weight bearing and gait parameters in patients with chronic stroke (Persian)]. Archives of Rehabilitation. 2016; 17(1):64-73.

[3] Soleimanzadeh Ardabili N, Vahdat I, Abdollahi I, Rostami M. [Evaluation of spasticity variations at the elbow joint of CVA patients according to the biomechanical indices (Persian)]. Archives of Rehabilitation. 2013; 14(3):96-106.

[4] Burridge JH, Wood DE, Taylor PN, McLellan DL. Indices to describe different muscle activation patterns, identified during treadmill walking, in people with spastic drop-foot. Medical Engineering \& Physics. 2001; 23(6):427-34. doi: 10.1016/s13504533(01)00061-3

[5] Deberg L, Taheri Andani M, Hosseinipour M, Elahinia M. An SMA passive ankle foot orthosis: Design, modeling, and experimental evaluation. Smart Materials Research. 2014; 1-11. doi: $10.1155 / 2014 / 572094$

[6] Lamkin-Kennard K. Design of a pneumatically actuated robotic assist device for patients with foot drop [ $\mathrm{PhD}$ dissertation]. Rochester: Rochester Institute of Technology; 2010.

[7] Laufer Y, Hausdorff JM, Ring H. Effects of a foot drop neuroprosthesis on functional abilities, social participation, and gait velocity. American Journal of Physical Medicine \& Rehabilitation. 2009; 88(1):14-20. doi: 10.1097/phm.0b013e3181911246

[8] Demneh ES. The effects of orthotics on the sensori-motor problems of the foot and ankle after stroke [PhD dissertation]. Salford: University of Salford; 2011.

[9] Guillebastre B, Calmels P, Rougier P. Effects of rgid and dynamic ankle-foot orthoses on normal gait. Foot \& Ankle International. 2009; 30(1):51-6. doi: 10.3113/fai.2009.0051

[10] Panwalkar N, Aruin AS. Role of ankle foot orthoses in the outcome of clinical tests of balance. Disability and Rehabilitation: Assistive Technology. 2012; 8(4):314-20. doi: 10.3109/17483107.2012.721158

[11] Leung J, Moseley A. Impact of ankle-foot orthoses on gait and leg muscle activity in adults with hemiplegia. Physiotherapy. 2003; 89(1):39-55. doi: 10.1016/s0031-9406(05)60668-2

[12] Schaekers L, Meuws L. What is the effect of an ankle-foot orthosis (AFO) on the dynamic balance and walking capacity in stroke patients [MSc. thesis]. Hasselt: University of Hasselt; 2014.

[13] Teasell RW, McRae MP, Foley N, Bhardwaj A. Physical and functional correlations of ankle-foot orthosis use in the rehabilitation of stroke patients. Archives of Physical Medicine and Rehabilitation. 2001; 82(8):1047-9. doi: 10.1053/apmr.2001.25078

[14] Mills K, Blanch P, Chapman AR, McPoil TG, Vicenzino B. Foot orthoses and gait: A systematic review and meta-analysis of literature pertaining to potential mechanisms. British Journal of Sports Medicine. 2009; 44(14):1035-46. doi: 10.1136/bjsm.2009.066977
[15] Guerra Padilla M, Molina Rueda F, Alguacil Diego IM. Effect of ankle-foot orthosis on postural control after stroke: A systematic review. Neurología (English Edition). 2014; 29(7):423-32. doi: 10.1016/j.nrleng.2011.10.014

[16] Tyson S, Sadeghi-Demneh E, Nester C. A systematic review and meta-analysis of the effect of an ankle-foot orthosis on gait biomechanics after stroke. Clinical Rehabilitation. 2013; 27(10):879_ 91. doi: $10.1177 / 0269215513486497$

[17] Alam M, Choudhury IA, Mamat AB. Mechanism and design analysis of articulated ankle foot orthoses for drop-foot. The Scientific World Journal. 2014; 1-14. doi: 10.1155/2014/867869

[18] Moher D. Preferred reporting items for systematic reviews and meta-analyses: The PRISMA statement. Annals of Internal Medicine. 2009; 151(4):264. doi: 10.7326/0003-4819-151-4 200908180-00135

[19] Burdett RG, Borello-France D, Blatchly C, Potter C. Gait comparison of subjects with hemiplegia walking unbraced, with ankle-foot orthosis, and with Air-Stirrup brace. Physical Therapy. 1988; 68(8):1197-203. doi: 10.1093/ptj/68.8.1197

[20] Chin R, Hsiao-Wecksler ET, Loth E, Kogler G, Manwaring SD, Tyson SN, et al. A pneumatic power harvesting ankle-foot orthosis to prevent foot-drop. Journal of NeuroEngineering and Rehabilitation. 2009; 6(1):19. doi: 10.1186/1743-0003-6-19

[21] Yokoyama O, Sashika H, Hagiwara A, Yamamoto S, Yasui T. Kinematic effects on gait of a newly designed ankle-foot orthosis with oil damper resistance: A case series of 2 patients with hemiplegia. Archives of Physical Medicine and Rehabilitation. 2005; 86(1):162-6. doi: 10.1016/j.apmr.2003.11.026

[22] Daher N, Lee S, Yang YJ. Effects of elastic band orthosis (aider) on balance and gait in chronic stroke patients. Physical Therapy Rehabilitation Science. 2013; 2(2):81-6. doi: 10.14474/ ptrs.2013.2.2.81

[23] Hale J, Seale J, Jennings J, DiBello T. An advanced ground reaction design ankle-foot orthosis to improve gait and balance in individuals with post-stroke hemiparesis. Journal of Prosthetics and Orthotics. 2013; 25(1):42-7. doi: 10.1097/jpo.0b013e31827ba11e

[24] Diamond MF, Ottenbacher KJ. Effect of a tone-inhibiting dynamic ankle-foot orthosis on stride characteristics of an adult with hemiparesis. Physical Therapy. 1990; 70(7):423-30. doi: $10.1093 / \mathrm{ptj} / 70.7 .423$

[25] Hesse S. Rehabilitation of gait after stroke. Topics in Geriatric Rehabilitation. 2003; 19(2):109-26. doi: 10.1097/00013614 200304000-00005

[26] Silver-Thorn B, Herrmann A, Current T, McGuire J. Effect of ankle orientation on heel loading and knee stability for post-stroke individuals wearing ankle-foot orthoses. Prosthetics and Orthotics International. 2011; 35(2):150-62. doi: 10.1177/0309364611399146

[27] Carse B, Bowers R, Meadows BC, Rowe P. The immediate effects of fitting and tuning solid ankle-foot orthoses in early stroke rehabilitation. Prosthetics and Orthotics International. 2015; 39(6):454-62. doi: 10.1177/0309364614538090

[28] Doğğan A, MengüllüoĞĞlu M, Özgirgin N. Evaluation of the effect of ankle-foot orthosis use on balance and mobility in hemi- 
paretic stroke patients. Disability and Rehabilitation. 2011; 33(1516):1433-9. doi: 10.3109/09638288.2010.533243

[29] Franceschini M, Massucci M, Ferrari L, Agosti M, Paroli C. Effects of an ankle-foot orthosis on spatiotemporal parameters and energy cost of hemiparetic gait. Clinical Rehabilitation. 2003; 17(4):368-72. doi: 10.1191/0269215503cr622oa

[30] Park JH, Chun MH, Ahn JS, Yu JY, Kang SH. Comparison of gait analysis between anterior and posterior ankle foot orthosis in hemiplegic patients. American Journal of Physical Medicine \& Rehabilitation. 2009; 88(8):630-4. doi: 10.1097/ phm.0b013e3181a9f30d

[31] Simons CDM, van Asseldonk EHF, Kooij H van der, Geurts $\mathrm{ACH}$, Buurke JH. Ankle-foot orthoses in stroke: Effects on functional balance, weight-bearing asymmetry and the contribution of each lower limb to balance control. Clinical Biomechanics. 2009; 24(9):769-75. doi: 10.1016/j.clinbiomech.2009.07.006

[32] Rao N, Chaudhuri G, Hasso D, D’Souza K, Wening J, Carlson C, et al. Gait assessment during the initial fitting of an ankle foot orthosis in individuals with stroke. Disability and Rehabilitation: Assistive Technology. 2008; 3(4):201-7. doi: 10.1080/17483100801973023

[33] Kobayashi T, Leung AKL, Akazawa Y, Hutchins SW. The effect of varying the plantarflexion resistance of an ankle-foot orthosis on knee joint kinematics in patients with stroke. Gait \& Posture. 2013; 37(3):457-9. doi: 10.1016/j.gaitpost.2012.07.028

[34] Lairamore C, Garrison MK, Bandy W, Zabel R. Comparison of tibialis anterior muscle electromyography, ankle angle, and velocity when individuals post stroke walk with different orthoses. Prosthetics and Orthotics International. 2011; 35(4):402-10. doi: $10.1177 / 0309364611417040$

[35] Jamshidi N, Rostami M, Najarian S, Bagher Menhaj M, Saadatnia M, Salamia F. Differences in center of pressure trajectory between normal and steppage gait. Journal of Research in Medical Sciences. 2010; 15(1):33-40. PMCID: PMC3082780

[36] Cruz TH, Dhaher YY. Impact of ankle-foot-orthosis on frontal plane behaviors post-stroke. Gait \& Posture. 2009; 30(3):312-6. doi: 10.1016/j.gaitpost.2009.05.018

[37] Wang RY, Lin PY, Lee CC, Yang YR. Gait and balance performance improvements attributable to ankle? Foot orthosis in subjects with hemiparesis. American Journal of Physical Medicine \& Rehabilitation. 2007; 86(7):556-62. doi: 10.1097/ phm.0b013e31806dd0d3

[38] Nolan KJ, Savalia KK, Yarossi M, Elovic EP. Evaluation of a dynamic ankle foot orthosis in hemiplegic gait: A case report. Neuro Rehabilitation. 2010; 27(4):343-50. doi: 10.3233/NRE2010-0618

[39] Bregman DJJ, De Groot V, Van Diggele P, Meulman H, Houdijk H, Harlaar J. Polypropylene ankle foot orthoses to overcome drop-foot gait in central neurological patients: A mechanical and functional evaluation. Prosthetics and Orthotics International. 2010; 34(3):293-304. doi: 10.3109/03093646.2010.495969 